\title{
Generalized model of interacting integrable tops
}

\section{A. Grekov, ${ }^{a, c, b, e}$ I. Sechin ${ }^{a, c, b}$ and A. Zotov ${ }^{a, b, d, e}$}

\author{
${ }^{a}$ Steklov Mathematical Institute of Russian Academy of Sciences, \\ Gubkina str. 8, Moscow 119991, Russia \\ ${ }^{b}$ ITEP, B. Cheremushkinskaya str. 25, Moscow 117218, Russia \\ ${ }^{c}$ Center for Advanced Studies, Skolkovo Institute of Science and Technology, \\ Nobel str. 1, Moscow 143026, Russia \\ ${ }^{d}$ National Research University Higher School of Economics, \\ Usacheva str. 6, Moscow 119048, Russia \\ ${ }^{e}$ Moscow Institute of Physics and Technology, \\ Inststitutskii per. 9, Dolgoprudny 141700, Moscow region, Russia \\ E-mail: grekovandrew@mail.ru, shnbuz@gmail.com, zotov@mi-ras.ru
}

ABSTRACT: We introduce a family of classical integrable systems describing dynamics of $M$ interacting $\mathrm{gl}_{N}$ integrable tops. It extends the previously known model of interacting elliptic tops. Our construction is based on the $\mathrm{GL}_{N} R$-matrix satisfying the associative Yang-Baxter equation. The obtained systems can be considered as extensions of the spin type Calogero-Moser models with (the classical analogues of) anisotropic spin exchange operators given in terms of the $R$-matrix data. In $N=1$ case the spin Calogero-Moser model is reproduced. Explicit expressions for $\mathrm{gl}_{N M}$-valued Lax pair with spectral parameter and its classical dynamical $r$-matrix are obtained. Possible applications are briefly discussed.

Keywords: Lattice Integrable Models, Matrix Models, Differential and Algebraic Geometry

ARXIV EPRINT: 1905.07820 


\section{Contents}

1 Introduction 1

2 Lax equations $\quad 6$

$2.1 \quad R$-matrix properties $\quad 6$

2.2 Lax pair and equations of motion $\quad 8$

2.3 Hamiltonian description 11

$\begin{array}{lll}2.4 & \text { Interacting tops } & 14\end{array}$

3 Classical $r$-matrix $\quad 15$

4 Examples $\quad 17$

$\begin{array}{lll}4.1 & \text { Elliptic models } & 17\end{array}$

$\begin{array}{lll}4.2 & \text { Trigonometric models } & 19\end{array}$

4.3 Rational models 20

$\begin{array}{lll}5 & \text { Discussion } & 21\end{array}$

$\begin{array}{ll}\text { A Definitions and identities } & 23\end{array}$

$\begin{array}{ll}\text { B Spin } \mathrm{gl}_{M} \text { Calogero-Moser model } & 24\end{array}$

$\begin{array}{ll}\text { C Integrable } \mathrm{gl}_{N} \text { tops } & 26\end{array}$

\section{Introduction}

In this paper we describe the classical integrable $\mathrm{gl}_{N M}$ model given by the Hamiltonian of the following form:

$$
\mathcal{H}=\sum_{i=1}^{M} \frac{p_{i}^{2}}{2}+\sum_{i=1}^{M} H^{\mathrm{top}}\left(\mathcal{S}^{i i}\right)+\frac{1}{2} \sum_{i, j: i \neq j}^{M} \mathcal{U}\left(\mathcal{S}^{i j}, \mathcal{S}^{j i}, q_{i}-q_{j}\right),
$$

where $p_{i}$ and $q_{j}$ are the canonical variables:

$$
\left\{p_{i}, q_{j}\right\}=\delta_{i j}, \quad\left\{p_{i}, p_{j}\right\}=\left\{q_{i}, q_{j}\right\}=0, \quad i, j=1 \ldots M .
$$

For all $i, j=1 \ldots M \mathcal{S}^{i j}$ are $N \times N$ matrices of "classical spin" variables, i.e.

$$
\mathcal{S}^{i j}=\sum_{a, b=1}^{N} \mathcal{S}_{a b}^{i j} e_{a b} \in \operatorname{Mat}(N, \mathbb{C}),
$$


where $\left\{e_{a b}, a, b=1 \ldots N\right\}$ is the standard basis in $\operatorname{Mat}(N, \mathbb{C})$. They are naturally arranged into $N M \times N M$ block-matrix $\mathcal{S}$ :

$$
\mathcal{S}=\sum_{i, j=1}^{M} E_{i j} \otimes \mathcal{S}^{i j}=\sum_{i, j=1}^{M} \sum_{a, b=1}^{N} \mathcal{S}_{a b}^{i j} E_{i j} \otimes e_{a b} \in \operatorname{Mat}(N M, \mathbb{C}),
$$

where $\left\{E_{i j}, i, j=1 \ldots M\right\}$ is the standard basis in $\operatorname{Mat}(M, \mathbb{C})$. The Poisson structure is given by the Poisson-Lie brackets on $\mathrm{gl}_{N M}^{*}$ Lie coalgebra:

$$
\left\{\mathcal{S}_{a b}^{i j}, \mathcal{S}_{c d}^{k l}\right\}=\mathcal{S}_{c b}^{k j} \delta^{i l} \delta_{a d}-\mathcal{S}_{a d}^{i l} \delta^{k j} \delta_{b c}
$$

Integrable tops. In order to clarify the structure of the Hamiltonian (1.1) consider the case $M=1$. Then the last term in (1.1) is absent, and we are left with a free particle (with momenta $\left.p_{1}\right)$ and the Hamiltonian $H^{\text {top }}\left(\mathcal{S}^{11}\right)$ of integrable top of Euler-Arnold type [3-7]. Here we deal with the models admitting the Lax pairs with spectral parameter on elliptic curves $[61,62]$. The general form for equations of motion (for the top like models) is

$$
\dot{S}=[S, J(S)],
$$

where $S \in \operatorname{Mat}(N, \mathbb{C})$ is the matrix of dynamical variables, while the inverse inertia tensor $J$ is a linear map

$$
J(S)=\sum_{i, j, k, l=1}^{N} J_{i j k l} e_{i j} S_{l k} \in \operatorname{Mat}(N, \mathbb{C}) .
$$

In the general case the model (1.6) is not integrable. It is integrable for some special $J(S)$ only. More precisely, here we consider special tops, which were described in $[1,36$, 37, 39-41, 68] for elliptic, trigonometric and rational cases respectively. All of them can be written $[40,41,44]$ in the $R$-matrix form based on a quantum $\mathrm{GL}_{N} R$-matrix (in the fundamental representation) satisfying the associative Yang-Baxter equation [25, 26, 54]:

$$
R_{12}^{\hbar}\left(q_{12}\right) R_{23}^{\eta}\left(q_{23}\right)=R_{13}^{\eta}\left(q_{13}\right) R_{12}^{\hbar-\eta}\left(q_{12}\right)+R_{23}^{\eta-\hbar}\left(q_{23}\right) R_{13}^{\hbar}\left(q_{13}\right), \quad q_{a b}=q_{a}-q_{b} .
$$

Having solution of (1.8) with some additional properties (see the next section) the inverse inertia tensor comes from the term $m_{12}(z)$ in the classical limit expansion:

$$
R_{12}^{\hbar}(z)=\frac{1}{\hbar} 1_{N} \otimes 1_{N}+r_{12}(z)+\hbar m_{12}(z)+O\left(\hbar^{2}\right) .
$$

Namely, for

$$
m_{12}(z)=\sum_{i, j, k, l=1}^{N} m_{i j k l}(z) e_{i j} \otimes e_{k l}
$$

the components of $J$ are

$$
J_{i j k l}=m_{i j k l}(0),
$$

that is

$$
J(S)=\operatorname{tr}_{2}\left(m_{12}(0) S_{2}\right), \quad S_{2}=1_{N} \otimes S .
$$


The Hamiltonian of the model is of the form:

$$
H^{\text {top }}(S)=\frac{1}{2} \operatorname{tr}(S J(S))=\frac{1}{2} \operatorname{tr}_{12}\left(m_{12}(0) S_{1} S_{2}\right), \quad S_{1}=S \otimes 1_{N} .
$$

This expression enters (1.1). The phase space of the model is a coadjoint orbit

$$
\mathcal{M}^{\text {top }}=\mathcal{O}_{N}
$$

of $\mathrm{GL}_{N}$ Lie group, i.e. the space spanned by $S_{i j}$ with some fixed eigenvalues of matrix $S$ (or the Casimir functions $C_{k}=\operatorname{tr} S^{k}$ ). Its dimension depends on the eigenvalues. The minimal orbit $\mathcal{O}_{N}^{\min }$ corresponds to $N-1$ coincident eigenvalues, i.e. the matrix $S$ (up to a matrix proportional to identity matrix) is of rank one:

$$
\operatorname{dim} \mathcal{O}_{N}^{\min }=2(N-1) .
$$

The Lax pair is given in the appendix $\mathrm{C}$.

Spin Calogero-Moser model. In the case $N=1$ the second term in (1.1) is trivial, and the last one boils down to the spin Calogero-Moser model [8-10, 27, 28]:

$$
H^{\text {spin }}=\sum_{i=1}^{M} \frac{p_{i}^{2}}{2}-\sum_{i>j}^{M} S_{i j} S_{j i} E_{2}\left(q_{i}-q_{j}\right),
$$

where $E_{2}(q)$ is the second Eisenstein function (A.4). Some details of the spin CalogeroMoser model are given in the appendix B. Let us only remark here that the model (1.16) is integrable through the Lax representation and the classical $r$-matrix structure on the constraints

$$
S_{i i}=\nu \text { for all } i=1 \ldots M
$$

supplemented by some gauge fixation conditions generated by the coadjoint action of the Cartan subgroup $\mathfrak{H}_{M} \subset \mathrm{GL}_{M}$. That is the phase space of the model is given by

$$
\mathcal{M}^{\text {spin }}=T^{*} \mathfrak{h}_{M} \times \mathcal{O}_{M} / / \mathfrak{H}_{M}
$$

where $\mathfrak{h}_{M}=\operatorname{Lie}\left(\mathfrak{H}_{M}\right)$ is the Lie algebra of $\mathfrak{H}_{M}$, and $\mathcal{O}_{M}$ is an orbit of the coadjoint action of $\mathrm{GL}_{M}$. The first factor in (1.18) describes the many-body degrees of freedom (1.2), and the second factor describes the "classical spin" variables. In the general case the spin variables can be parameterized by the set of canonically conjugated variables:

$$
\begin{aligned}
S_{i j} & =\sum_{a=1}^{N} \xi_{a}^{i} \eta_{a}^{j}, \\
\left\{\xi_{a}^{i}, \eta_{b}^{j}\right\} & =\delta_{a b} \delta_{i j}, \quad i, j=1 \ldots M, \quad a, b=1 \ldots N .
\end{aligned}
$$

The Poisson structure (B.7) is reproduced in this way. Using these notations it is easy to see that

$$
S_{i j} S_{j i}=\sum_{a, b=1}^{N} \xi_{a}^{i} \eta_{a}^{j} \xi_{b}^{j} \eta_{b}^{i}=\operatorname{tr}\left(\mathcal{S}^{i i} \mathcal{S}^{j j}\right),
$$


and the potential in the Hamiltonian (1.16) takes the form

$$
\mathcal{V}^{\text {spin }}\left(\mathcal{S}^{i i}, \mathcal{S}^{j j}, q_{i j}\right)=-\operatorname{tr}\left(\mathcal{S}^{i i} \mathcal{S}^{j j}\right) E_{2}\left(q_{i}-q_{j}\right) .
$$

Below we construct anisotropic (in $\operatorname{Mat}(N, \mathbb{C})$ space) generalizations of (1.21).

In the special case, when the matrix of spin variables $S$ is of rank 1 (it is the minimal $\left.\mathcal{O}_{M}^{\min } \operatorname{orbit}(1.15)\right)$

$$
S_{i j}=\xi_{i} \eta_{j}
$$

the reduction with respect to the action of $\mathfrak{H}_{M}$ leads to the spinless Calogero-Moser (CM) model $[15-19,38]$ since the second factor in (1.18) become trivial. Indeed, plugging (1.22) into (1.16) and using (1.17) we get

$$
H^{\mathrm{spin}}=\sum_{i=1}^{M} \frac{p_{i}^{2}}{2}-\nu^{2} \sum_{i>j}^{M} E_{2}\left(q_{i}-q_{j}\right) .
$$

The spinless Calogero-Moser models are gauge equivalent to the special top with the minimal orbit (1.15). See [1, 36, 37, 39] for details.

Interacting tops. Turning back to the $\mathrm{gl}_{N M}$ model (1.1) consider the special case when the matrix $\mathcal{S}$ is of rank 1:

$$
\mathcal{S}_{a b}^{i j}=\xi_{a}^{i} \eta_{b}^{j}
$$

We will see that in this case the last term in (1.1) is rewritten in the form

$$
\mathcal{U}\left(\mathcal{S}^{i j}, \mathcal{S}^{j i}, q_{i}-q_{j}\right)=\mathcal{V}\left(\mathcal{S}^{i i}, \mathcal{S}^{j j}, q_{i}-q_{j}\right),
$$

and the Hamiltonian (1.1) acquires the form

$$
\mathcal{H}^{\mathrm{tops}}=\sum_{i=1}^{M} \frac{p_{i}^{2}}{2}+\sum_{i=1}^{M} H^{\mathrm{top}}\left(\mathcal{S}^{i i}\right)+\frac{1}{2} \sum_{i, j: i \neq j}^{M} \mathcal{V}\left(\mathcal{S}^{i i}, \mathcal{S}^{j j}, q_{i}-q_{j}\right) .
$$

It describes mechanics of $M$ interacting integrable $\mathrm{gl}_{N}$ tops. The Hamiltonian of (1.26) type was introduced by A.P. Polychronakos [55-57] from his study of matrix models. Then the elliptic version of model (1.1) and (1.26) was described as $\mathrm{gl}_{N M}$ Hitchin system $[49,67,68]$ (see some details in section 4), and (1.1) was also generalized for arbitrary complex Lie group [45-47].

Similarly to the spin Calogero-Moser model the general model (1.1) requires additional constraints (cf. (1.17))

$$
\operatorname{tr}\left(\mathcal{S}^{i i}\right)=\nu \text { for all } i=1 \ldots M .
$$

They should be supplied with some gauge fixation conditions generated by the coadjoint action of $\mathfrak{H}_{N M}^{\prime} \subset \mathfrak{H}_{N M}$ - subgroup of the Cartan subgroup $\mathfrak{H}_{N M} \subset \mathrm{GL}_{N M}$ with elements of the form $\sum_{i=1}^{M} h_{i} E_{i i} \otimes 1_{N}$. Together with (1.27) the gauge fixation conditions are the second class constraints, and one can perform the Dirac reduction procedure to compute the final Poisson structure starting from the linear one (1.5). The phase space of the general model (1.1) is of the from:

$$
\mathcal{M}^{\text {gen }}=T^{*} \mathfrak{h}_{N M}^{\prime} \times \mathcal{O}_{N M} / / \mathfrak{H}_{N M}^{\prime}, \quad \mathfrak{h}_{N M}^{\prime}=\operatorname{Lie}\left(\mathfrak{H}_{N M}^{\prime}\right) .
$$


For the interacting tops case $(1.24)-(1.26)$ the orbit $\mathcal{O}_{N M}$ becomes $\mathcal{O}_{N M}^{\min }$. Then the phase space

$$
\mathcal{M}^{\text {tops }}=T^{*} \mathfrak{h}_{N M}^{\prime} \times \mathcal{O}_{N M}^{\min } / / \mathfrak{H}_{N M}^{\prime}
$$

has dimension $2 N M$, while its "spin part" is of dimension

$$
\operatorname{dim}\left(\mathcal{O}_{N M}^{\min } / / \mathfrak{H}_{N M}^{\prime}\right)=2 N M-2 M .
$$

A brief summary of the described models is given in the following scheme:

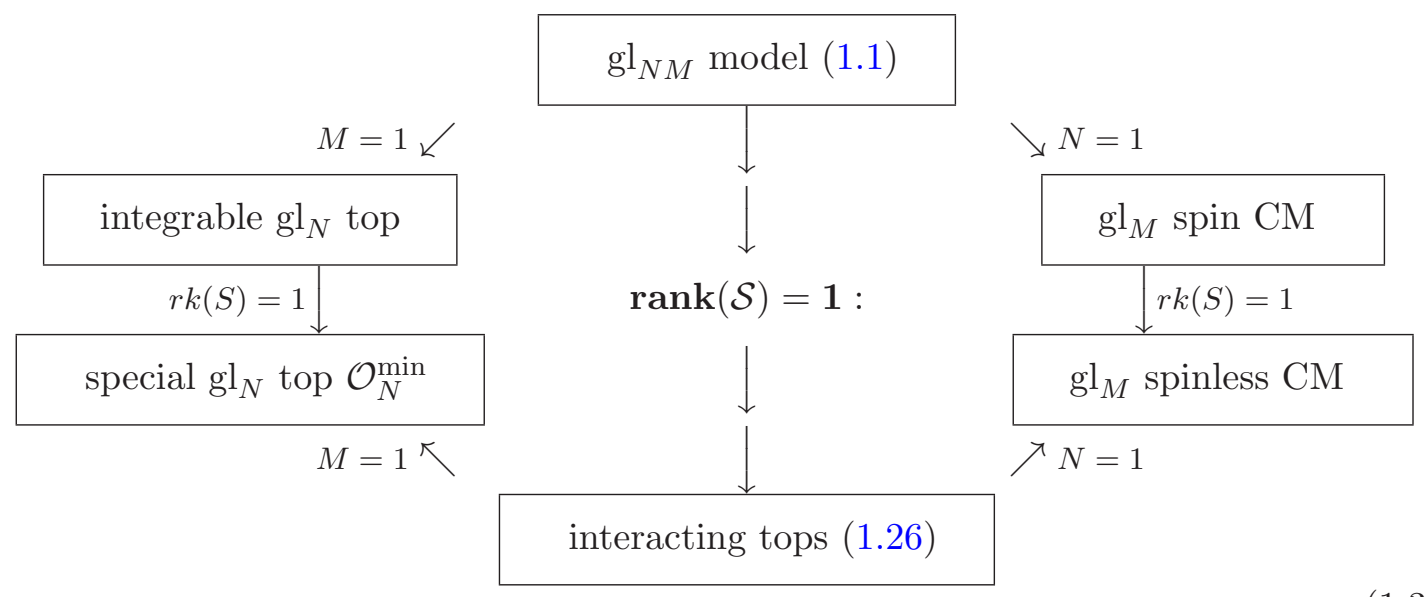

Purpose of the paper. is to describe a family of the models (1.1) and (1.26) in terms of $R$-matrices satisfying the associative Yang-Baxter equation (1.8). We give explicit formulae for $N M \times N M$ Lax pair with spectral parameter (see the next section) and compute the Hamiltonians (1.1) and (1.26). As a result we obtain the potentials

$$
\mathcal{U}\left(\mathcal{S}^{i j}, \mathcal{S}^{j i}, q_{i}-q_{j}\right)=\operatorname{tr}_{12}\left(\partial_{q_{i}} r_{21}\left(q_{i j}\right) P_{12} \mathcal{S}_{1}^{i j} \mathcal{S}_{2}^{j i}\right)
$$

for the general model (1.1) and

$$
\mathcal{V}\left(\mathcal{S}^{i i}, \mathcal{S}^{j j}, q_{i}-q_{j}\right)=\operatorname{tr}_{12}\left(\partial_{q_{i}} r_{12}\left(q_{i j}\right) \mathcal{S}_{1}^{i i} \mathcal{S}_{2}^{j j}\right)
$$

for the model of interacting tops (1.26). Notice that in the simplest case related to the rational Yang's XXX $R$-matrix

$$
R_{12}^{z}\left(q_{i j}\right)=\frac{1_{N} \otimes 1_{N}}{z}+\frac{P_{12}}{q_{i}-q_{j}}
$$

we get just the spin Calogero-Moser model written in terms of matrix variables:

$$
\mathcal{V}=-\frac{\operatorname{tr}\left(\mathcal{S}^{i i} \mathcal{S}^{j j}\right)}{\left(q_{i}-q_{j}\right)^{2}}
$$

Next, we proceed to the classical (dynamical) $r$-matrix. It is similar to the one for the spin Calogero-Moser case [8-10] but this time its matrix elements are $R$-matrices themselves. The classical exchange relations are verified directly. This guarantees the Poisson commutativity of the Hamiltonians generated by the Lax matrix. 
The answers (1.32) and (1.33) depend on the classical $r$-matrix, which appears from the quantum one in the limit (1.9). The quantum $R$-matrix enters the higher Hamiltonians. It should satisfy a set of properties which we discuss in the next section. The most general $R$ matrix satisfying all the required properties is the elliptic Baxter-Balavin's one. In this case the integrable models are known. They were first described by Polychronakos in [55-57] and later reproduced as Hitchin type systems on the bundles with nontrivial characteristic classes in $[49,67]$.

The family of the obtained models includes new integrable systems in the trigonometric and rational cases. While the quantization of the potential $\mathcal{V}$ from (1.35) is given by isotropic spin exchange operator $\hat{\mathcal{V}}=-P_{i j} /\left(q_{i}-q_{j}\right)^{2}$, the obtained general answer (1.32)(1.33) leads to the anisotropic potentials. An example of such anisotropic extension to the spin (trigonometric) Calogero-Moser-Sutherland model was first suggested by Hikami and Wadati [35] at quantum level. From the point of view of (1.33) their answer corresponds to the $\mathrm{gl}_{2} \mathrm{XXZ} r$-matrix. At the same time the set of trigonometric $R$-matrices satisfying the required properties is much lager $[2,58,59]$, and all these $R$-matrices can be used for construction of the integrable tops [36, 37]. The results of the present paper are also valid for all these cases. An example based on the $\mathrm{gl}_{2}$ 7-th vertex deformation of the XXZ $R$-matrix is given section 4 . Similarly, in the rational case the admissible $R$-matrices includes not only the Yang's $R$-matrix (1.34) but also its deformations such as 11 -vertex $R$ matrix [20] and its higher rank versions [40,41]. An example related to 11-vertex $R$-matrix is given in section 4 .

Possible applications of the described models are discussed in the end. Namely, we argue that the obtained models can be used for construction of higher Hamiltonians for the anisotropic generalizations of the Haldane-Shastry-Inozemtsev long-range spin chains. The latter is important for the proof of integrability of these chains, which still remains an open problem.

\section{Lax equations}

In this section we construct the $N M \times N M$ Lax pair $\mathcal{L}(z), \mathcal{M}(z)$ satisfying the Lax equations

$$
\dot{\mathcal{L}}(z)=[\mathcal{L}(z), \mathcal{M}(z)]
$$

for the model (1.1). Our construction is based on $\mathrm{GL}_{N} R$-matrix - solution of the associative Yang-Baxter equation (1.8). Besides (1.8) the $R$-matrix should also satisfy a set of properties.

\section{$2.1 \quad R$-matrix properties}

We consider $R$-matrices satisfying (1.8) and (1.9). Let us also impose the following set of conditions for $\mathrm{GL}_{N} R$-matrices under consideration:

Expansion near $z=0$ :

$$
R_{12}^{\hbar}(z)=\frac{1}{z} P_{12}+R_{12}^{\hbar,(0)}+z R_{12}^{\hbar,(1)}+O\left(z^{2}\right)
$$


Also,

$$
R_{12}^{z,(0)}=\frac{1}{z} 1_{N} \otimes 1_{N}+r_{12}^{(0)}+O(z), \quad r_{12}(z)=\frac{1}{z} P_{12}+r_{12}^{(0)}+z r_{12}^{(1)}+O\left(z^{2}\right) .
$$

Skew-symmetry: ${ }^{1}$

$$
R_{12}^{\hbar}(z)=-R_{21}^{-\hbar}(-z)=-P_{12} R_{12}^{-\hbar}(-z) P_{12}, \quad P_{12}=\sum_{i, j=1}^{N} E_{i j} \otimes E_{j i} .
$$

Unitarity:

$$
R_{12}^{\hbar}(z) R_{21}^{\hbar}(-z)=f^{\hbar}(z) 1_{N} \otimes 1_{N}, \quad f^{\hbar}(z)=\wp(\hbar)-\wp(z) .
$$

We are also going to use the Fourier symmetry:

$$
R_{12}^{\hbar}(z) P_{12}=R_{12}^{z}(\hbar)
$$

It is not necessary but convenient property. The following relations on the coefficients of expansions (1.9) and (2.2) follow from the skew-symmetry:

$$
\begin{aligned}
r_{12}(z) & =-r_{21}(-z), & m_{12}(z) & =m_{21}(-z), \\
R_{12}^{\hbar,(0)} & =-R_{21}^{-\hbar,(0)}, & r_{12}^{(0)} & =-r_{21}^{(0)} .
\end{aligned}
$$

Similarly, from the Fourier symmetry we have (see details in [69]):

$$
\begin{aligned}
R_{12}^{z,(0)} & =r_{12}(z) P_{12}, \\
R_{12}^{z,(1)} & =m_{12}(z) P_{12}, \\
r_{12}^{(0)} & =r_{12}^{(0)} P_{12} .
\end{aligned}
$$

In what follows we use special notation for the $R$-matrix derivative:

$$
F_{12}^{z}(q)=\partial_{q} R_{12}^{z}(q)
$$

It is the $R$-matrix analogue of the function (A.5) entering the $M$-matrix of the spin Calogero-Moser model (B.3) likewise $R$-matrix itself is a matrix analogue of the Kronecker function (A.1) due to similarity of (A.6) and (1.8). See [42, 43]. Then from the classical limit (1.9) we have

$$
F_{12}^{0}(q)=\left.\partial_{q} R_{12}^{z}(q)\right|_{z=0}=\partial_{q} r_{12}(q) .
$$

The latter is the $R$-matrix analogue of the function $-E_{2}(q)$ (A.12) entering the CalogeroMoser potential. Notice also that $F_{12}^{0}(q)=F_{21}^{0}(-q)$ due to (2.7). From (2.9) and (2.2) the local expansion near $q=0$ is as follows

$$
F_{12}^{z}(q)=-\frac{1}{q^{2}} P_{12}+R_{12}^{z,(1)}+O(q)
$$

\footnotetext{
${ }^{1} P_{12}$ entering (2.4) is the permutation operator, $\left(P_{12}\right)^{2}=1_{N} \otimes 1_{N}$.
} 
and, therefore,

$$
F_{12}^{0}(q)=-\frac{1}{q^{2}} P_{12}+\left.R_{12}^{z,(1)}\right|_{z=0}+O(q) \stackrel{(2.8)}{=}-\frac{1}{q^{2}} P_{12}+m_{12}(0) P_{12}+O(q) .
$$

On the other hand

$$
F_{12}^{0}(q) \stackrel{(2.10)}{=} \partial_{q} r_{12}(q) \stackrel{(2.3)}{=}-\frac{1}{q^{2}} P_{12}+r_{12}^{(1)}+O(q) .
$$

From (2.12) and (2.13) we conclude that

$$
r_{12}^{(1)}=m_{12}(0) P_{12} .
$$

In the elliptic case the set of properties is fulfilled by the Baxter-Belavin [11-13] $R$ matrix (C.14). A family of trigonometric $R$-matrices include the XXZ 6-vertex one, its 7 -vertex deformation [20] and $\mathrm{GL}_{N}$ generalizations [2, 58, 59]. See a brief review and applications to integrable tops in $[36,37]$. The rational $R$-matrices possessing the properties are the XXX Yang's $R$-matrix, its 11-vertex deformation [20] and higher rank analogues obtained from the elliptic case by special limiting procedure [66]. The final answer for such $R$-matrix was obtained in $[40,41]$ through the gauge equivalence between the relativistic top with minimal orbit and the rational Ruijsenaars-Schneider model.

\subsection{Lax pair and equations of motion}

Using coefficients of the expansion of the $\mathrm{GL}_{N} R$-matrix near $z=0$ we define $N M \times N M$ Lax pair

$$
\begin{aligned}
L(z) & =\sum_{i, j=1}^{M} E_{i j} \otimes L^{i j}(z), \quad L^{i j}(z) \in \operatorname{Mat}_{N} \quad L(z) \in \operatorname{Mat}_{N M}, \\
L^{i j}(z) & =\delta_{i j}\left(p_{i} 1_{N}+\operatorname{tr}_{2}\left(\mathcal{S}_{2}^{i i} R_{12}^{z,(0)} P_{12}\right)\right)+\left(1-\delta_{i j}\right) \operatorname{tr}_{2}\left(\mathcal{S}_{2}^{i j} R_{12}^{z}\left(q_{i j}\right) P_{12}\right),
\end{aligned}
$$

and similarly for $M^{i j}(z) \in \operatorname{Mat}_{N}$

$$
M^{i j}(z)=\delta_{i j} \operatorname{tr}_{2}\left(\mathcal{S}_{2}^{i i} R_{12}^{z,(1)} P_{12}\right)+\left(1-\delta_{i j}\right) \operatorname{tr}_{2}\left(\mathcal{S}_{2}^{i j} F_{12}^{z}\left(q_{i j}\right) P_{12}\right) .
$$

where the entries are defined from (2.2) and (2.9). The tensor notations are similar to those used in (C.3)-(C.6).

Proposition 2.1. Consider an R-matrix satisfying the associative Yang-Baxter equation (1.8), the classical limit (1.9) and the set of properties from the previous paragraph. Then the Lax equation (2.1) holds true for the Lax pair (2.15)-(2.17) on the constraints

$$
\operatorname{tr}\left(\mathcal{S}^{i i}\right)=\text { const. }, \forall i
$$

(cf. (4.4)) and provides the following equations of motion for off-diagonal $N \times N$ blocks of $\mathcal{S}$ :

$$
\begin{aligned}
\dot{\mathcal{S}}^{i j}= & \sum_{k: k \neq i, j}^{M}\left(\mathcal{S}^{i k} \operatorname{tr}_{2}\left(\mathcal{S}_{2}^{k j} F_{12}^{0}\left(q_{k j}\right) P_{12}\right)-\operatorname{tr}_{2}\left(\mathcal{S}_{2}^{i k} F_{12}^{0}\left(q_{i k}\right) P_{12}\right) \mathcal{S}^{k j}\right)+ \\
& +\mathcal{S}^{i i} \operatorname{tr}_{2}\left(\mathcal{S}_{2}^{i j} F_{12}^{0}\left(q_{i j}\right) P_{12}\right)-\operatorname{tr}_{2}\left(\mathcal{S}_{2}^{i i} m_{12}(0)\right) \mathcal{S}^{i j}- \\
& -\operatorname{tr}_{2}\left(\mathcal{S}_{2}^{i j} F_{12}^{0}\left(q_{i j}\right) P_{12}\right) \mathcal{S}^{j j}+\mathcal{S}^{i j} \operatorname{tr}_{2}\left(\mathcal{S}_{2}^{j j} m_{12}(0)\right)
\end{aligned}
$$


for diagonal $N \times N$ blocks of $\mathcal{S}$ :

$$
\dot{\mathcal{S}}^{i i}=\left[\mathcal{S}^{i i}, \operatorname{tr}_{2}\left(m_{12}(0) \mathcal{S}_{2}^{i i}\right)\right]+\sum_{k: k \neq i}^{M}\left(\mathcal{S}^{i k} \operatorname{tr}_{2}\left(\mathcal{S}_{2}^{k i} F_{21}^{0}\left(q_{i k}\right) P_{12}\right)-\operatorname{tr}_{2}\left(\mathcal{S}_{2}^{i k} F_{12}^{0}\left(q_{i k}\right) P_{12}\right) \mathcal{S}^{k i}\right),
$$

and for momenta:

$$
\dot{p}_{i}=-\sum_{k: k \neq i}^{M} \operatorname{tr}_{23}\left(\partial_{q_{i}} F_{32}^{0}\left(q_{i k}\right) P_{23} \mathcal{S}_{2}^{i k} \mathcal{S}_{3}^{k i}\right) .
$$

Proof. We imply $p_{i}=\dot{q}_{i}$ in the formulae above. This follows from the Hamiltonian description, which is given in the next paragraph.

1. Let us begin with the non-diagonal blocks. Consider the one numbered $i j(i \neq j)$. The 1.h.s. of the Lax equations reads

$$
\text { l.h.s. }=\dot{\mathcal{L}}^{i j}(z)=\operatorname{tr}_{2}\left(\dot{\mathcal{S}}_{2}^{i j} R_{12}^{z}\left(q_{i j}\right) P_{12}\right)+\operatorname{tr}_{2}\left(\mathcal{S}_{2}^{i j} F_{12}^{z}\left(q_{i j}\right) P_{12}\right)\left(\dot{q}_{i}-\dot{q}_{j}\right) .
$$

The r.h.s. of the Lax equation is as follows:

$$
\text { r.h.s. }=\mathcal{L}^{i j} \mathcal{M}^{j j}-\mathcal{M}^{i i} \mathcal{L}^{i j}+\mathcal{L}^{i i} \mathcal{M}^{i j}-\mathcal{M}^{i j} \mathcal{L}^{j j}+\sum_{k: k \neq i, j}^{M}\left(\mathcal{L}^{i k} \mathcal{M}^{k j}-\mathcal{M}^{i k} \mathcal{L}^{k j}\right) .
$$

The last sum is computed using identity

$$
R_{12}^{z}(x) F_{23}^{z}(y)-F_{12}^{z}(x) R_{23}^{z}(y)=F_{23}^{0}(y) R_{13}^{z}(x+y)-R_{13}^{z}(x+y) F_{12}^{0}(x),
$$

which follows from (1.8). It is the $R$-matrix analogue of (A.7). In its turn (A.7) is the key tool underlying ansatz for the Lax pairs with spectral parameter [38]. For $k \neq i, j$ we have

$$
\begin{aligned}
\mathcal{L}^{i k} \mathcal{M}^{k j}-\mathcal{M}^{i k} \mathcal{L}^{k j}= & \\
& =\operatorname{tr}_{23}\left(R_{12}^{z}\left(q_{i k}\right) P_{12} \mathcal{S}_{2}^{i k} F_{13}^{z}\left(q_{k j}\right) P_{13} \mathcal{S}_{3}^{k j}\right)-\operatorname{tr}_{23}\left(F_{12}^{z}\left(q_{i k}\right) P_{12} \mathcal{S}_{2}^{i k} R_{13}^{z}\left(q_{k j}\right) P_{13} \mathcal{S}_{3}^{k j}\right)= \\
& =\operatorname{tr}_{23}\left(\left(R_{12}^{z}\left(q_{i k}\right) F_{23}^{z}\left(q_{k j}\right)-F_{12}^{z}\left(q_{i k}\right) R_{23}^{z}\left(q_{k j}\right)\right) P_{12} P_{13} \mathcal{S}_{2}^{i k} \mathcal{S}_{3}^{k j}\right) \stackrel{(2.24)}{=} \\
& =\operatorname{tr}_{23}\left(\left(F_{23}^{0}\left(q_{k j}\right) R_{13}^{z}\left(q_{i j}\right)-R_{13}^{z}\left(q_{i j}\right) F_{12}^{0}\left(q_{i k}\right)\right) P_{12} P_{13} \mathcal{S}_{2}^{i k} \mathcal{S}_{3}^{k j}\right)= \\
& =\operatorname{tr}_{23}\left(R_{12}^{z}\left(q_{i j}\right) P_{12}\left(\mathcal{S}_{2}^{i k} \mathcal{S}_{3}^{k j} F_{23}^{0}\left(q_{k j}\right) P_{23}-F_{23}^{0}\left(q_{i k}\right) P_{23} \mathcal{S}_{3}^{i k} \mathcal{S}_{2}^{k j}\right)\right) .
\end{aligned}
$$

This expression provides the upper line in the equations of motion (2.19). To proceed we need degenerations of the identity (2.24) when $y \rightarrow 0$. It comes from the expansions (2.2), (2.11) and (2.13):

$$
R_{12}^{z}(x) R_{23}^{z,(1)}-F_{12}^{z}(x) R_{23}^{z,(0)}=r_{23}^{(1)} R_{13}^{z}(x)-R_{13}^{z}(x) F_{12}^{0}(x)-\frac{1}{2} P_{23} \partial_{x}^{2} R_{13}^{z}(x) .
$$

In the same way in the limit $x \rightarrow 0$ (2.24) takes the form

$$
R_{12}^{z,(0)} F_{23}^{z}(y)-R_{12}^{z,(1)} R_{23}^{z}(y)=F_{23}^{0}(y) R_{13}^{z}(y)-R_{13}^{z}(y) r_{12}^{(1)}+\frac{1}{2} \partial_{y}^{2} R_{13}^{z}(y) P_{12} .
$$


Similarly to the ordinary (spin) Calogero-Moser case the terms linear in momenta in the r.h.s. $(2.23)\left(p_{i}-p_{j}\right) \mathcal{M}^{i j}$ are cancelled out by the last term in the l.h.s. of (2.22). Consider the first and the fourth terms from (2.23) without momenta. Using evaluations similar to $(2.25)$ we get

$$
\begin{aligned}
\mathcal{L}^{i j} \mathcal{M}^{j j}-\mathcal{M}^{i j}\left(\mathcal{L}^{j j}-p_{j} 1_{N}\right)= \\
=\operatorname{tr}_{23}\left(\left(R_{12}^{z}\left(q_{i j}\right) R_{23}^{z,(1)}-F_{12}^{z}\left(q_{i j}\right) R_{23}^{z,(0)}\right) P_{12} P_{13} \mathcal{S}_{2}^{i j} \mathcal{S}_{3}^{j j}\right) \stackrel{(2.26)}{=} \\
=\operatorname{tr}_{23}\left(\left(r_{23}^{(1)} R_{13}^{z}\left(q_{i j}\right)-R_{13}^{z}\left(q_{i j}\right) F_{12}^{0}\left(q_{i j}\right)-\frac{1}{2} P_{23} \partial_{q_{i}}^{2} R_{13}^{z}\left(q_{i j}\right)\right) P_{12} P_{13} \mathcal{S}_{2}^{i j} \mathcal{S}_{3}^{j j}\right)= \\
=\operatorname{tr}_{23}\left(R_{12}^{z}\left(q_{i j}\right) P_{12} \mathcal{S}_{2}^{i j} \mathcal{S}_{3}^{j j} m_{23}(0)\right)-\operatorname{tr}_{23}\left(R_{12}^{z}\left(q_{i j}\right) P_{12} F_{23}^{0}\left(q_{i j}\right) P_{23} \mathcal{S}_{3}^{i j} \mathcal{S}_{2}^{j j}\right)- \\
\quad-\frac{1}{2} \operatorname{tr}_{23}\left(\partial_{q_{i}}^{2} R_{12}^{z}\left(q_{i j}\right) P_{12} \mathcal{S}_{2}^{i j} \mathcal{S}_{3}^{j j}\right),
\end{aligned}
$$

where the relation (2.14) was also used (for the first term in the answer). The first and the second terms in the obtained answer provide the last line in the equations of motion (2.19), while the last term in (2.19) is the "unwanted term".

In the same way, using (2.27) one gets

$$
\begin{aligned}
\left(\mathcal{L}^{i i}-\right. & \left.p_{i} 1_{N}\right) \mathcal{M}^{i j}-\mathcal{M}^{i i} \mathcal{L}^{i j}= \\
= & \operatorname{tr}_{23}\left(R_{12}^{z}\left(q_{i j}\right) P_{12} \mathcal{S}_{2}^{i i} \mathcal{S}_{3}^{i j} F_{23}^{0}\left(q_{i j}\right) P_{23}\right)-\operatorname{tr}_{23}\left(R_{12}^{z}\left(q_{i j}\right) P_{12} m_{23}(0) \mathcal{S}_{3}^{i i} \mathcal{S}_{2}^{i j}\right)+ \\
& +\frac{1}{2} \operatorname{tr}_{23}\left(\partial_{q_{i}}^{2} R_{12}^{z}\left(q_{i j}\right) P_{12} \mathcal{S}_{3}^{i i} \mathcal{S}_{2}^{i j}\right)
\end{aligned}
$$

Again, the first two terms provide an input to equations of motion - the second line in (2.19). The last term is the "unwanted term". It is cancelled by the one from (2.28) after taking the trace over the third component and imposing the constraints (2.18).

2. Consider a diagonal $N \times N$ block (numbered $i i$ ) of the Lax equation. The l.h.s. of the Lax equations is

$$
\text { l.h.s. }=\dot{\mathcal{L}}^{i i}(z)=\dot{p}_{i} 1_{N}+\operatorname{tr}_{2}\left(\dot{\mathcal{S}}_{2}^{i i} R_{12}^{z,(0)} P_{12}\right) \stackrel{(2.8)}{=} \dot{p}_{i} 1_{N}+\operatorname{tr}_{2}\left(\dot{\mathcal{S}}_{2}^{i i} r_{12}(z)\right) \text {. }
$$

The r.h.s. of the Lax equation is as follows:

$$
\text { r.h.s. }=\left[\mathcal{L}^{i i}, \mathcal{M}^{i i}\right]+\sum_{k: k \neq i}^{M}\left(\mathcal{L}^{i k} \mathcal{M}^{k i}-\mathcal{M}^{i k} \mathcal{L}^{k i}\right) \text {. }
$$

The commutator term in (2.31) provides the commutator term in the equations of motion (2.20) since it is the input from the internal $i i$-th top's dynamics, and this was derived in [44]. See (C.2)-(C.4). In order to simplify expression in the sum we need the following degeneration of (1.8):

$$
R_{12}^{z}(x) R_{23}^{z}(y)=R_{13}^{z}(x+y) r_{12}(x)+r_{23}(y) R_{13}^{z}(x+y)-\partial_{z} R_{13}^{z}(x+y),
$$


It corresponds to $\hbar=\eta=z$. In the scalar case it is the identity (A.9). In the limit $x=q=-y$ from $(2.32)$ we get

$$
R_{12}^{z}(q) R_{23}^{z}(-q)=R_{13}^{z,(0)} r_{12}(q)-r_{32}(q) R_{13}^{z,(0)}-\partial_{z} R_{13}^{z,(0)}+F_{32}^{0}(q) P_{13},
$$

or, using (2.8)

$$
R_{12}^{z}(q) R_{23}^{z}(-q)=\left(r_{13}(z) r_{32}(q)-r_{32}(q) r_{13}(z)\right) P_{13}-F_{13}^{0}(z) P_{13}+F_{32}^{0}(q) P_{13} .
$$

By differentiating (2.34) with respect to $q$ we obtain

$$
R_{12}^{z}(q) F_{23}^{z}(-q)-F_{12}^{z}(q) R_{23}^{z}(-q)=\left[F_{32}^{0}(q), r_{13}(z)\right] P_{13}-\partial_{q} F_{32}^{0}(q) P_{13} .
$$

For $k \neq i$ consider

$$
\begin{aligned}
& \mathcal{L}^{i k} \mathcal{M}^{k i}-\mathcal{M}^{i k} \mathcal{L}^{k i}= \\
& \quad=\operatorname{tr}_{23}\left(\left(R_{12}^{z}\left(q_{i k}\right) F_{23}^{z}\left(q_{k i}\right)-F_{12}^{z}\left(q_{i k}\right) R_{23}^{z}\left(q_{k i}\right)\right) P_{12} P_{13} \mathcal{S}_{2}^{i k} \mathcal{S}_{3}^{k i}\right) \stackrel{(2.35)}{=} \\
& \quad=\operatorname{tr}_{23}\left(\left(\left[F_{32}^{0}\left(q_{i k}\right), r_{13}(z)\right] P_{13}-\partial_{q_{i}} F_{32}^{0}\left(q_{i k}\right) P_{13}\right) P_{12} P_{13} \mathcal{S}_{2}^{i k} \mathcal{S}_{3}^{k i}\right)
\end{aligned}
$$

The commutator term in the obtained expression yields the sum term in the equations of motion (2.20), while the last term in (2.36) provides equations of motion (2.21). Indeed,

$$
\operatorname{tr}_{23}\left(\left(\partial_{q_{i}} F_{32}^{0}\left(q_{i k}\right) P_{13}\right) P_{12} P_{13} \mathcal{S}_{2}^{i k} \mathcal{S}_{3}^{k i}\right)=1_{N} \operatorname{tr}_{23}\left(\partial_{q_{i}} F_{32}^{0}\left(q_{i k}\right) P_{23} \mathcal{S}_{2}^{i k} \mathcal{S}_{3}^{k i}\right)
$$

and the momenta is the scalar component in the l.h.s. (2.30).

\subsection{Hamiltonian description}

The Hamiltonian function. Let us compute the Hamiltonian for the model (2.15)(2.21). It comes from the generating function

$$
\frac{1}{2 N} \operatorname{tr}\left(\mathcal{L}^{2}(z)\right)=\frac{1}{2 N} \sum_{i=1}^{M} \operatorname{tr}\left(\mathcal{L}^{i i}(z)\right)^{2}+\frac{1}{2 N} \sum_{i \neq j}^{M} \operatorname{tr}\left(\mathcal{L}^{i j}(z) \mathcal{L}^{j i}(z)\right) .
$$

Consider

$$
\operatorname{tr}\left(\mathcal{L}^{i i}(z)\right)^{2}=N p_{i}^{2}+2 p_{i} \operatorname{tr}_{12}\left(r_{12}(z) \mathcal{S}_{2}^{i i}\right)+\operatorname{tr}_{123}\left(r_{12}(z) r_{13}(z) \mathcal{S}_{2}^{i i} \mathcal{S}_{3}^{i i}\right)
$$

As before, the numbered tensor components are $\operatorname{Mat}(N, \mathbb{C})$-valued. In order to simplify (2.39) we use the identity (see $[42,43]$ )

$$
r_{12}(z) r_{13}(z+w)-r_{23}(w) r_{12}(z)+r_{13}(z+w) r_{23}(w)=m_{12}(z)+m_{23}(w)+m_{13}(z+w)
$$


which can be treated as a half of the classical Yang-Baxter equation. ${ }^{2}$ In the limit $w \rightarrow$ 0 (2.40) yields

$$
r_{12}(z) r_{13}(z)=r_{23}^{(0)} r_{12}(z)-r_{13}(z) r_{23}^{(0)}-F_{13}^{0}(z) P_{23}+m_{12}(z)+m_{23}(0)+m_{13}(z) .
$$

Also, we are going to use the following $R$-matrix property:

$$
\operatorname{tr}_{1} R_{12}^{q}(z)=\operatorname{tr}_{2} R_{12}^{q}(z)=\tilde{\phi}(z, q) 1_{N}
$$

where $\tilde{\phi}(z, q)$ is the Kronecker function (A.1) but with possibly different normalization factor and normalization of arguments. The property (2.42) holds true in the elliptic case (C.15) as well as for its trigonometric and rational degenerations. From (2.42), expansion (1.9) and (A.10) we also have similar properties for $\operatorname{tr}_{1} r_{12}(z)=\tilde{E}_{1}(z)$ and $\operatorname{tr}_{1} m_{12}(z)$ - they are scalar operators:

$$
\operatorname{tr}_{1} R_{12}^{q}(z)=q^{-1} 1_{N}+\operatorname{tr}_{1} r_{12}(z)+q \operatorname{tr}_{1} m_{12}(z)+O\left(q^{2}\right) .
$$

Return now to (2.39). On the constraints (2.18) the second term is equal to $2 p_{i} \tilde{E}_{1}(z)$ const. After summation over $i$ it provides the Hamiltonian proportional to $\sum_{i=1}^{M} p_{i}$. Plugging (2.41) into the last term of (2.39) we get

$$
\begin{aligned}
& \operatorname{tr}_{123}\left(r_{12}(z) r_{13}(z) \mathcal{S}_{2}^{i i} \mathcal{S}_{3}^{i i}\right)= \\
& \quad=\operatorname{tr}_{123}\left(\left(r_{23}^{(0)} r_{12}(z)-r_{13}(z) r_{23}^{(0)}-F_{13}^{0}(z) P_{23}+m_{23}(0)+m_{12}(z)+m_{13}(z)\right) \mathcal{S}_{2}^{i i} \mathcal{S}_{3}^{i i}\right) .
\end{aligned}
$$

Due to (2.42) the first two terms are cancelled out after taking the trace over the component 1. By the same reason the last two terms in (2.44) provide $2 \operatorname{tr}_{1}\left(m_{12}(z)\right) \operatorname{tr}_{23}\left(\mathcal{S}_{2}^{i i} \mathcal{S}_{3}^{i i}\right)$. These are constants on the constraints (2.18). The rest of the terms are

$$
\operatorname{tr}_{123}\left(\left(-F_{13}(z) P_{23}+m_{23}(0)\right) \mathcal{S}_{2}^{i i} \mathcal{S}_{3}^{i i}\right) \stackrel{(2.42)}{=} \tilde{E}_{2}(z) \operatorname{tr}\left(\mathcal{S}^{i i}\right)^{2}+N \operatorname{tr}_{23}\left(m_{23}(0) \mathcal{S}_{2}^{i i} \mathcal{S}_{3}^{i i}\right)
$$

where $\tilde{E}_{2}(z) 1_{N}=-\operatorname{tr}_{1}\left(F_{13}^{0}(z)\right)=-\partial_{z} \operatorname{tr}_{1}\left(r_{13}(z)\right)=-\partial_{z} \tilde{E}_{1}(z) 1_{N}$. It is a scalar function coming from (2.43) and similar to $E_{2}(z)$ (A.4). The factor $N$ in the last term comes from $\operatorname{tr}_{1}$. The first term in (2.45) is a part of the Casimir function $\operatorname{tr} \mathcal{S}^{2}$, and the second one is $H^{\text {top }}\left(\mathcal{S}^{i i}\right)$ from $(1.1)$ :

$$
H^{\mathrm{top}}\left(\mathcal{S}^{i i}\right)=\frac{1}{2} \operatorname{tr}_{12}\left(m_{12}(0) \mathcal{S}_{1}^{i i} \mathcal{S}_{2}^{i i}\right) .
$$

Next, consider

$$
\begin{aligned}
\operatorname{tr}\left(\mathcal{L}^{i j}(z) \mathcal{L}^{j i}(z)\right) & =\operatorname{tr}_{123}\left(R_{12}^{z}\left(q_{i j}\right) P_{12} R_{13}^{z}\left(q_{j i}\right) P_{13} \mathcal{S}_{2}^{i j} \mathcal{S}_{3}^{j i}\right)= \\
& =\operatorname{tr}_{123}\left(R_{12}^{z}\left(q_{i j}\right) R_{23}^{z}\left(q_{j i}\right) P_{12} P_{13} \mathcal{S}_{2}^{i j} \mathcal{S}_{3}^{j i}\right) \stackrel{(2.34)}{=} \\
& =\operatorname{tr}_{123}\left(\left(\left[r_{13}(z), r_{32}\left(q_{i j}\right)\right]-F_{13}^{0}(z)+F_{32}^{0}\left(q_{i j}\right)\right) P_{23} \mathcal{S}_{2}^{i j} \mathcal{S}_{3}^{j i}\right)
\end{aligned}
$$

\footnotetext{
${ }^{2}$ The difference of two such equations gives the classical Yang-Baxter equation for the classical $r$-matrix.
} 
Again, the commutator term vanishes after taking the trace over the first tensor component. Therefore,

$$
\begin{aligned}
\operatorname{tr}\left(\mathcal{L}^{i j}(z) \mathcal{L}^{j i}(z)\right) & =\operatorname{tr}_{123}\left(\left(-F_{13}^{0}(z)+F_{32}^{0}\left(q_{i j}\right)\right) P_{23} \mathcal{S}_{2}^{i j} \mathcal{S}_{3}^{j i}\right)= \\
& =\tilde{E}_{2}(z) \operatorname{tr}\left(\mathcal{S}^{i j} \mathcal{S}^{j i}\right)+N \operatorname{tr}_{12}\left(F_{21}^{0}\left(q_{i j}\right) P_{12} \mathcal{S}_{1}^{i j} \mathcal{S}_{2}^{j i}\right)
\end{aligned}
$$

Finally, for the potential term from (1.1) we have

$$
\mathcal{U}\left(\mathcal{S}^{i j}, \mathcal{S}^{j i}, q_{i j}\right)=\operatorname{tr}_{12}\left(F_{21}^{0}\left(q_{i j}\right) P_{12} \mathcal{S}_{1}^{i j} \mathcal{S}_{2}^{j i}\right)
$$

and the Hamiltonian (1.1) is of the form:

$$
\mathcal{H}=\sum_{i=1}^{M} \frac{p_{i}^{2}}{2}+\frac{1}{2} \sum_{i=1}^{M} \operatorname{tr}_{12}\left(m_{12}(0) \mathcal{S}_{1}^{i i} \mathcal{S}_{2}^{i i}\right)+\sum_{i<j}^{M} \operatorname{tr}_{12}\left(F_{21}^{0}\left(q_{i j}\right) P_{12} \mathcal{S}_{1}^{i j} \mathcal{S}_{2}^{j i}\right)
$$

In $M=1$ case $\mathcal{H}$ reproduce the Hamiltonian of the integrable top, while in the $M=1$ case we obtain the spin Calogero-Moser Hamiltonian (1.16) up to terms containing $S_{i i}$ they are constant in this case (1.17).

Poisson brackets. The Poisson structure (before reduction (1.28)) consists of the canonical brackets for positions and momenta

$$
\left\{p_{i}, q_{j}\right\}=\delta_{i j}, \quad\left\{p_{i}, p_{j}\right\}=\left\{q_{i}, q_{j}\right\}=0, \quad i=1 \ldots M
$$

and the linear Poisson-Lie brackets for the $\mathcal{S}$ variables. They are of the form (B.7) but for $\operatorname{Mat}(N M, \mathbb{C})$ case instead of $\operatorname{Mat}(M, \mathbb{C})$ in $(\mathrm{B} .7)$. It is convenient to write down these brackets in terms of $\operatorname{Mat}(N, \mathbb{C})$-valued blocks $\mathcal{S}^{i j}$. For $i, j, k, l=1 \ldots M$ and $a, b, c, d=$ $1 \ldots N$ :

$$
\left\{\mathcal{S}_{a b}^{i j}, \mathcal{S}_{c d}^{k l}\right\}=\mathcal{S}_{c b}^{k j} \delta^{i l} \delta_{a d}-\mathcal{S}_{a d}^{i l} \delta^{k j} \delta_{b c}
$$

or

$$
\left\{\mathcal{S}_{1}^{i j}, \mathcal{S}_{2}^{k l}\right\}=P_{12} \mathcal{S}_{1}^{k j} \delta^{i l}-\mathcal{S}_{1}^{i l} P_{12} \delta^{k j}
$$

where $P_{12}$ as before the permutation operator in $\operatorname{Mat}(N, \mathbb{C})^{\otimes 2}$. For the diagonal blocks we have

$$
\left\{\mathcal{S}_{1}^{i i}, \mathcal{S}_{2}^{k k}\right\}=\left[P_{12}, \mathcal{S}_{1}^{i i}\right] \delta^{i k}
$$

It is verified directly that

Proposition 2.2. The Poisson structure (2.51), (2.53) and the Hamiltonian (2.50) provides equations of (2.19)-(2.21), i.e. for the l.h.s. of the Lax equation (2.1) we have

$$
\dot{\mathcal{L}}(z)=\{\mathcal{H}, \mathcal{L}(z)\}
$$




\subsection{Interacting tops}

Suppose the matrix $\mathcal{S}$ is of rank one, i.e. (1.24) is fulfilled. Consider the potential

$$
\operatorname{tr}_{12}\left(F_{21}^{0}\left(q_{i j}\right) P_{12} \mathcal{S}_{1}^{i j} \mathcal{S}_{2}^{j i}\right)=\sum_{a, b, c, d=1}^{N}\left(F_{12}^{0}\left(q_{j i}\right) P_{12}\right)_{a b, c d} \mathcal{S}_{b a}^{i j} \mathcal{S}_{d c}^{j i} .
$$

The right multiplication of an element $T_{12}=\sum_{i, j, k, l=1}^{N} T_{i j k l} E_{i j} \otimes E_{k l} \in \operatorname{Mat}(N, \mathbb{C})^{\otimes 2}$ by permutation operator $P_{12}$ yields $T_{i j k l} \rightarrow T_{i l k j}$, i.e.

$$
\operatorname{tr}_{23}\left(F_{32}^{0}\left(q_{i j}\right) P_{23} \mathcal{S}_{2}^{i j} \mathcal{S}_{3}^{j i}\right)=\sum_{a, b, c, d=1}^{N}\left(F_{12}^{0}\left(q_{j i}\right)\right)_{a d, c b} \mathcal{S}_{b a}^{i j} \mathcal{S}_{d c}^{j i} .
$$

In the rank 1 case we have

$$
\mathcal{S}_{b a}^{i j} \mathcal{S}_{d c}^{j i}=\xi_{b}^{i} \eta_{a}^{j} \xi_{d}^{j} \eta_{c}^{i}=\mathcal{S}_{b c}^{i i} \mathcal{S}_{d a}^{j j}
$$

Therefore,

$$
\operatorname{tr}_{23}\left(F_{32}^{0}\left(q_{i j}\right) P_{23} \mathcal{S}_{2}^{i j} \mathcal{S}_{3}^{j i}\right)=\operatorname{tr}_{12}\left(F_{12}^{0}\left(q_{j i}\right) \mathcal{S}_{1}^{j j} \mathcal{S}_{2}^{i i}\right)=\operatorname{tr}_{12}\left(F_{12}^{0}\left(q_{i j}\right) \mathcal{S}_{1}^{i i} \mathcal{S}_{2}^{j j}\right) .
$$

The Hamiltonian of interacting tops model acquires the form:

$$
\mathcal{H}^{\text {tops }}=\sum_{i=1}^{M} \frac{p_{i}^{2}}{2}+\frac{1}{2} \sum_{i=1}^{M} \operatorname{tr}_{12}\left(m_{12}(0) \mathcal{S}_{1}^{i i} \mathcal{S}_{2}^{i i}\right)+\sum_{i<j}^{M} \operatorname{tr}_{12}\left(F_{12}^{0}\left(q_{i j}\right) \mathcal{S}_{1}^{i i} \mathcal{S}_{2}^{j j}\right) \text {. }
$$

From the Poisson brackets (2.51), (2.54) we get the corresponding equations of motion:

$$
\begin{aligned}
\dot{\mathcal{S}}^{i i} & =\left[\mathcal{S}^{i i}, \operatorname{tr}_{2}\left(m_{12}(0) \mathcal{S}_{2}^{i i}\right)\right]+\sum_{k: k \neq i}^{M}\left[\mathcal{S}^{i i}, \operatorname{tr}_{2}\left(F_{12}^{0}\left(q_{i k}\right) \mathcal{S}_{2}^{k k}\right)\right], \\
\dot{p}_{i} & =-\sum_{k: k \neq i}^{M} \operatorname{tr}_{12}\left(\partial_{q_{i}} F_{12}^{0}\left(q_{i k}\right) \mathcal{S}_{1}^{i i} \mathcal{S}_{2}^{k k}\right) .
\end{aligned}
$$

In this model we are left with $M$ matrix variables $\mathcal{S}^{i i} \in \operatorname{Mat}(N, \mathbb{C})$ of rank one. It is notable that the spin part of the phase space (1.29) is isomorphic to a product of $M$ minimal coadjoint orbits (1.15):

$$
\mathcal{O}_{N M}^{\min } / / \mathfrak{H}_{N M}^{\prime} \cong \underbrace{O_{N}^{\min } \times \ldots \times O_{N}^{\min }}_{M \text { times }} .
$$

Notice that the orbits $O_{N}^{\min }$ come from the constraints conditions (2.18). Hence it appears that

1. For the model of interacting tops the constraints (2.18) play the role of fixation of the Casimir functions for $M$ copies of $\mathrm{gl}_{N}^{*}$ (of rank one). Consequently, equations of motion (2.61) are not changed after reduction. For the $N=1$ case (the spin CalogeroMoser model) we get $\dot{S}_{i i}=0$ since the r.h.s. of (2.61) consists of commutators. 
2. The model of interacting tops is formulated in terms of $M \operatorname{Mat}(N, \mathbb{C})$-valued variables of rank one, describing the minimal coadjoint orbits. The integrability condition is that all Casimir functions $\operatorname{tr}\left(\mathcal{S}^{i i}\right)$ are equal to each other. ${ }^{3}$

3. The spin part of the phase space for the model of interacting tops coincides with the phase space of $\mathrm{GL}_{N}$ classical spin chain on $M$ sites with the spins described by minimal coadjoint orbits at each site.

Let us also remark that the top like models with matrix-valued variables were studied in $[44,69]$ and [14]. In contrast to these papers here we deal with the models, where the matrix variables have their own internal dynamics.

\section{Classical $r$-matrix}

In this section we describe the classical $r$-matrix structure for the Lax matrix (2.16). Since $\mathcal{L} \in \operatorname{Mat}(N M, \mathbb{C})$ then the corresponding classical $\mathrm{gl}_{N M} r$-matrix $\mathbf{r} \in \operatorname{Mat}(N M, \mathbb{C})^{\otimes 2}$. Recall that for the Lax matrix we use the matrix basis $(2.15)$, in which $\mathcal{L} \in \operatorname{Mat}(M, \mathbb{C}) \otimes$ $\operatorname{Mat}(N, \mathbb{C})$. Let the $\operatorname{Mat}(M, \mathbb{C})$-valued tensor components be numbered by primed numbers, and the $\operatorname{Mat}(N, \mathbb{C})$-valued components - without primes (as before). Introduce the following $r$-matrix:

$$
\mathbf{r}_{1^{\prime} 2^{\prime} 12}(z, w)=\sum_{i=1}^{M}{\stackrel{1^{\prime}}{E}}_{i i} \otimes{\stackrel{2^{\prime}}{E}}_{i i} \otimes r_{12}(z-w)+\sum_{i \neq j}^{M}{\stackrel{1^{\prime}}{E}}_{i j} \otimes{\stackrel{2^{\prime}}{E}}_{j i} \otimes R_{12}^{z-w}\left(q_{i j}\right) P_{12}
$$

so that $\mathbf{r}_{1^{\prime} 2^{\prime} 12} \in \operatorname{Mat}(M, \mathbb{C})^{\otimes 2} \otimes \operatorname{Mat}(N, \mathbb{C})^{\otimes 2}$. In the case $M=1$ we come to a nondynamical $r$-matrix describing the top model, while in the $N=1$ we reproduce the dynamical $r$-matrix of the spin Calogero-Moser model (B.9). $r$-matrices of these type are known in $\mathrm{gl}_{N M}$ case and can be extended for arbitrary complex semisimple Lie algebras [22-24, 48]. In the elliptic case $(3.1)$ is known in the quantum case as well [49]. At the same time (3.1) includes the cases, which have not been described yet. For instance, the new cases correspond to the rational $R_{12}^{z}(q)$-matrix from [40, 41]. Similarly to the Lax equations the construction of the $r$-matrix (3.1) is based on the associative Yang-Baxter equation (1.8) and its degenerations.

Proposition 3.1. Consider an R-matrix satisfying the associative Yang-Baxter equation (1.8), the classical limit (1.9) and the set of properties from the section 2.1. Then for the Lax pair (2.15)-(2.16) the following classical exchange relation holds true:

$$
\begin{aligned}
\left\{\mathcal{L}_{1^{\prime} 1}(z), \mathcal{L}_{2^{\prime} 2}(w)\right\}= & {\left[\mathcal{L}_{1^{\prime} 1}(z), \mathbf{r}_{1^{\prime} 2^{\prime} 12}(z, w)\right]-\left[\mathcal{L}_{2^{\prime} 2}(w), \mathbf{r}_{2^{\prime} 1^{\prime} 21}(w, z)\right]-} \\
& -\sum_{k=1}^{M} \operatorname{tr}\left(\mathcal{S}^{k k}\right) \partial_{q_{k}} \mathbf{r}_{1^{\prime} 2^{\prime} 12}(z, w)
\end{aligned}
$$

\footnotetext{
${ }^{3}$ More precisely, we can not confirm that the model is not integrable in the case $\operatorname{tr}\left(\mathcal{S}^{i i}\right) \neq \operatorname{tr}\left(\mathcal{S}^{j j}\right)$, but the presented Lax pair does not work in this case.
} 
where

$$
\begin{gathered}
\mathcal{L}_{1^{\prime} 1}(z)=\sum_{i, j=1}^{M} E_{i j} \otimes 1_{M} \otimes \mathcal{L}^{i j}(z) \otimes 1_{N}, \\
\mathcal{L}_{2^{\prime} 2}(w)=\sum_{k, l=1}^{M} 1_{M} \otimes E_{k l} \otimes 1_{N} \otimes \mathcal{L}^{k l}(w) .
\end{gathered}
$$

The Poisson brackets in the l.h.s. of (3.2) are given by (2.51)-(2.54).

Proof. The proof is direct. Let us demonstrate how to verify (3.2) for several components of $\stackrel{1}{E}_{i j} \otimes{\stackrel{2}{E^{\prime}}}_{k l}$, which are similar to those considered in (B.11)-(B.16) for the spin CalogeroMoser model.

the tensor component ${\stackrel{1}{E_{i j}^{\prime}}}_{i} \otimes{\stackrel{2}{E^{\prime}}}_{j k}(i \neq j, j \neq k, i \neq k)$ :

1.h.s. of (3.2):

$$
\operatorname{tr}_{34}\left(R_{13}^{z}\left(q_{i j}\right) P_{13} R_{24}^{w}\left(q_{j k}\right) P_{24}\left\{\mathcal{S}_{3}^{i j}, \mathcal{S}_{4}^{j k}\right\}\right)=-\operatorname{tr}_{3}\left(\mathcal{S}_{3}^{i k} R_{23}^{w}\left(q_{j k}\right) P_{23} R_{13}^{z}\left(q_{i j}\right) P_{13}\right) .
$$

r.h.s. of (3.2):

$$
\operatorname{tr}_{3}\left(\mathcal{S}_{3}^{i k}\left(R_{13}^{z}\left(q_{i k}\right) P_{13} R_{12}^{z-w}\left(q_{k j}\right) P_{12}-R_{12}^{z-w}\left(q_{i j}\right) P_{12} R_{23}^{w}\left(q_{i k}\right) P_{23}\right)\right) .
$$

Expressions (3.5) and (3.6) coincide due to (1.8) and (2.4).

the tensor component ${\stackrel{1}{E_{i i}^{\prime}}}_{i} \otimes{\stackrel{2}{E^{\prime}}}_{i j}(i \neq j)$ :

l.h.s. of (3.2):

$$
\begin{array}{r}
\operatorname{tr}_{4}\left(\left\{p_{i}, R_{24}^{w}\left(q_{i j}\right)\right\} P_{24} \mathcal{S}_{4}^{i j}\right)+\operatorname{tr}_{34}\left(r_{13}(z) R_{24}^{w}\left(q_{i j}\right) P_{24}\left\{\mathcal{S}_{3}^{i i}, \mathcal{S}_{4}^{i j}\right\}\right)= \\
=\operatorname{tr}_{3}\left(\mathcal{S}_{3}^{i j} \partial_{q_{i}} R_{23}^{w}\left(q_{i j}\right) P_{23}\right)-\operatorname{tr}_{3}\left(\mathcal{S}_{3}^{i j} R_{23}^{w}\left(q_{i j}\right) P_{23} r_{13}(z)\right) .
\end{array}
$$

r.h.s. of (3.2):

$$
\operatorname{tr}_{3}\left(\mathcal{S}_{3}^{i j}\left(R_{13}^{w}\left(q_{i j}\right) P_{13} R_{12}^{z-w}\left(q_{j i}\right) P_{12}-r_{12}(z-w) R_{23}^{w}\left(q_{i j}\right) P_{23}\right)\right) .
$$

Expressions (3.7) and (3.8) coincide due to (2.32) rewritten through the Fourier symmetry (2.6) as

$$
R_{a c}^{q_{i j}}(z) R_{b c}^{q_{j i}}(w)=-R_{a b}^{q_{i j}}(z-w) r_{a c}(z)+r_{b c}(w) R_{a b}^{q_{i j}}(z-w)-\partial_{q_{i}} R_{a b}^{q_{i j}}(z-w)
$$

for distinct $a, b, c$.

the tensor component ${\stackrel{1}{E_{i j}}}_{i} \otimes{\stackrel{2}{E^{\prime}}}_{j i}(i \neq j)$ :

l.h.s. of (3.2):

$$
\operatorname{tr}_{3}\left(\mathcal{S}_{3}^{j j} R_{13}^{z}\left(q_{i j}\right) P_{13} R_{23}^{w}\left(q_{j i}\right) P_{23}-\mathcal{S}_{3}^{i i} R_{23}^{w}\left(q_{j i}\right) P_{23} R_{13}^{z}\left(q_{i j}\right) P_{13}\right) .
$$


r.h.s. of $(3.2)$ :

$$
\begin{aligned}
\operatorname{tr}_{3}\left(\mathcal{S}_{3}^{i i}\left(r_{13}(z) R_{12}^{z-w}\left(q_{i j}\right) P_{12}-R_{12}^{z-w}\left(q_{i j}\right) P_{12} r_{23}(w)\right)\right)+ \\
\quad+\operatorname{tr}_{3}\left(\mathcal{S}_{3}^{j j}\left(-R_{12}^{z-w}\left(q_{i j}\right) P_{12} r_{13}(z)+r_{23}(w) R_{12}^{z-w}\left(q_{i j}\right) P_{12}\right)\right)- \\
\quad-\operatorname{tr}_{3}\left(\left(\mathcal{S}_{3}^{i i}-\mathcal{S}_{3}^{j j}\right) \partial_{q_{i}} R_{12}^{z-w}\left(q_{i j}\right) P_{12}\right) .
\end{aligned}
$$

The last term comes from the second line of (3.2). Again, expressions (3.10) and (3.11) coincide due to (3.9).

The rest of the components are verified similarly.

\section{Examples}

\subsection{Elliptic models}

Let us begin with the elliptic model $[29,30,49,67]$. The Lax pair is of the form:

$$
\mathcal{L}(z)=\sum_{i, j=1}^{M} E_{i j} \otimes \mathcal{L}^{i j}(z), \quad \mathcal{L}^{i j}(z) \in \operatorname{Mat}_{N} \quad \mathcal{L}(z) \in \operatorname{Mat}_{N M},
$$

where

$$
\begin{array}{r}
\mathcal{L}^{i j}(z)=\delta_{i j}\left(p_{i} 1_{N}+\mathcal{S}_{(0,0)}^{i i} 1_{N} E_{1}(z)+\sum_{\alpha \neq 0} \mathcal{S}_{\alpha}^{i i} T_{\alpha} \varphi_{\alpha}\left(z, \omega_{\alpha}\right)\right)+ \\
+\left(1-\delta_{i j}\right) \sum_{\alpha} \mathcal{S}_{\alpha}^{i j} T_{\alpha} \varphi_{\alpha}\left(z, \omega_{\alpha}+\frac{q_{i j}}{N}\right),
\end{array}
$$

where the basis $(\mathrm{C} .8)$ in $\operatorname{Mat}(N, \mathbb{C})$ is used. Similarly, the $M$-matrix is of the form

$$
\begin{array}{r}
\mathcal{M}^{i j}(z)=\delta_{i j} \mathcal{S}_{(0,0)}^{i i} \frac{E_{1}^{2}(z)-\wp(z)}{2 N} 1_{N}+\frac{1}{N} \delta_{i j} \sum_{\alpha \neq 0} \mathcal{S}_{\alpha}^{i i} T_{\alpha} f_{\alpha}\left(z, \omega_{\alpha}\right)+ \\
+\frac{1}{N}\left(1-\delta_{i j}\right) \sum_{\alpha} \mathcal{S}_{\alpha}^{i j} T_{\alpha} f_{\alpha}\left(z, \omega_{\alpha}+\frac{q_{i j}}{N}\right) .
\end{array}
$$

These formulae can be obtained from (2.15)-(2.17) and the $R$-matrix (C.15) together with (A.8).

The Lax equations hold on the constraints

$$
\mathcal{S}_{(0,0)}^{i i}=\text { const }, \quad \forall i .
$$

Instead of the standard basis (1.3) here we use the basis (C.8) for each $N \times N$ block. Then the Poisson structure (1.5) takes the form

$$
\left\{\mathcal{S}_{\alpha}^{i j}, \mathcal{S}_{\beta}^{k l}\right\}=\delta_{i l} \kappa_{\alpha, \beta} \mathcal{S}_{\alpha+\beta}^{k j}-\delta_{k j} \kappa_{\beta, \alpha} \mathcal{S}_{\alpha+\beta}^{i l},
$$

where $\kappa_{\alpha, \beta}$ are the constants from (C.10). 
The Hamiltonian easily follows from $\frac{1}{2 N} \operatorname{tr} \mathcal{L}^{2}(z)=\frac{1}{2 N} E_{2}(z) \operatorname{tr}\left(\mathcal{S}^{2}\right)+\mathcal{H}$ due to (C.11) and (A.8):

$$
\mathcal{H}=\frac{1}{2} \sum_{i=1}^{M} p_{i}^{2}-\frac{1}{2} \sum_{i=1}^{M} \sum_{\alpha \neq 0} \mathcal{S}_{\alpha}^{i i} \mathcal{S}_{-\alpha}^{i i} E_{2}\left(\omega_{\alpha}\right)-\frac{1}{2} \sum_{i \neq j}^{M} \sum_{\alpha} \mathcal{S}_{\alpha}^{i j} \mathcal{S}_{-\alpha}^{j i} E_{2}\left(\omega_{\alpha}+\frac{q_{i j}}{N}\right) .
$$

Let us show how this Hamiltonian is reproduced from the general formula (2.50). In order to get the second term in (4.6) one should substitute $m_{12}(0)$ into (2.50) from (C.17) and use relation (A.12). For evaluation of the last sum in (2.50) we need to calculate $F_{12}^{0}(q) P_{12}$. The answer for $F_{12}^{0}(q)$ is given in (C.18). Multiply it by $P_{12}=(1 / N) \sum_{b} T_{b} \otimes T_{-b}$ from the left:

$$
\begin{aligned}
F_{12}^{0}(q) P_{12}= & -\frac{1}{N^{2}} E_{2}(q) \sum_{b} T_{b} \otimes T_{-b} \\
& +\frac{1}{N^{2}} \sum_{a \neq(0,0) ; b} \varphi_{a}\left(q, \omega_{a}\right)\left(E_{1}\left(q+\omega_{a}\right)-E_{1}(q)+2 \pi \imath \partial_{\tau} \omega_{\alpha}\right) \kappa_{a, b}^{2} T_{a+b} \otimes T_{-a-b} .
\end{aligned}
$$

Let us redefine the summation index $b \rightarrow b-a$ in the last sum. Since $\kappa_{a, b}=\kappa_{a, b-a}$ we have

$$
\begin{aligned}
& F_{12}^{0}(q) P_{12}= \\
& \quad \frac{1}{N^{2}} \sum_{b} T_{b} \otimes T_{-b}\left(-E_{2}(q)+\sum_{a \neq 0 ; b} \varphi_{a}\left(q, \omega_{a}\right)\left(E_{1}\left(q+\omega_{a}\right)-E_{1}(q)+2 \pi \imath \partial_{\tau} \omega_{\alpha}\right) \kappa_{a, b}^{2}\right) \stackrel{(\mathrm{C} .22)}{=} \\
& \quad=-\frac{1}{N^{2}} \sum_{b} T_{b} \otimes T_{-b} E_{2}\left(\omega_{b}+\frac{q}{N}\right) .
\end{aligned}
$$

Finally,

$$
\operatorname{tr}_{12}\left(F_{21}^{0}\left(q_{i j}\right) P_{12} \mathcal{S}_{1}^{i j} \mathcal{S}_{2}^{j i}\right)=\sum_{\alpha} \mathcal{S}_{\alpha}^{i j} \mathcal{S}_{-\alpha}^{j i} E_{2}\left(\omega_{\alpha}+\frac{q_{i j}}{N}\right) .
$$

In the rank 1 case the answer for the Hamiltonian is given by (2.60). Plugging (C.18) into (2.60) we get

$$
\begin{aligned}
\mathcal{H}^{\text {tops }}= & \frac{1}{2} \sum_{i=1}^{M} p_{i}^{2}-\frac{1}{2} \sum_{i=1}^{M} \sum_{\alpha \neq 0} \mathcal{S}_{\alpha}^{i i} \mathcal{S}_{-\alpha}^{i i} E_{2}\left(\omega_{\alpha}\right)- \\
& -\frac{N}{2} \sum_{i \neq j}^{M}\left(E_{2}\left(q_{i j}\right) \mathcal{S}_{0}^{i i} \mathcal{S}_{0}^{j j}-\sum_{a \neq 0} \varphi_{a}\left(q_{i j}, \omega_{a}\right)\left(E_{1}\left(q_{i j}+\omega_{a}\right)-E_{1}\left(q_{i j}\right)+2 \pi \imath \partial_{\tau} \omega_{\alpha}\right) \mathcal{S}_{-\alpha}^{i i} \mathcal{S}_{\alpha}^{j j}\right) .
\end{aligned}
$$

Let us show how the latter expression appears from (4.6). In the rank one case using (C.11) (so that $\mathcal{S}_{\alpha}^{i j}=\operatorname{tr}\left(\mathcal{S}^{i j} T_{-\alpha}\right) / N$ ) we get

$$
\mathcal{S}_{\alpha}^{i j} \mathcal{S}_{-\alpha}^{j i}=\frac{\operatorname{tr}\left(\eta^{j} T_{-\alpha} \xi^{i}\right) \operatorname{tr}\left(\eta^{i} T_{\alpha} \xi^{j}\right)}{N^{2}}=\frac{\operatorname{tr}\left(\eta^{j} T_{-\alpha} \xi^{i} \eta^{i} T_{\alpha} \xi^{j}\right)}{N^{2}}=\frac{\operatorname{tr}\left(\mathcal{S}^{i i} T_{\alpha} \mathcal{S}^{j j} T_{-\alpha}\right)}{N^{2}} .
$$


In this way the Hamiltonian (4.6) acquires the form

$$
\begin{aligned}
\mathcal{H}^{\text {tops }}= & \frac{1}{2} \sum_{i=1}^{M} p_{i}^{2}-\frac{1}{2} \sum_{i=1}^{M} \sum_{\alpha \neq 0} \mathcal{S}_{\alpha}^{i i} \mathcal{S}_{-\alpha}^{i i} E_{2}\left(\omega_{\alpha}\right)- \\
& -\frac{1}{2} \sum_{i \neq j}^{M} \sum_{\alpha} \frac{\operatorname{tr}\left(\mathcal{S}^{i i} T_{\alpha} \mathcal{S}^{j j} T_{-\alpha}\right)}{N^{2}} E_{2}\left(\omega_{\alpha}+\frac{q_{i j}}{N}\right),
\end{aligned}
$$

which is the model of interacting tops of (1.26) type. The last terms in (4.12) can be simplified in the following way. Substitute $\mathcal{S}^{i i}=\sum_{\gamma} \mathcal{S}_{\gamma}^{i i} T_{\gamma}$ and $\mathcal{S}^{j j}=\sum_{\gamma} \mathcal{S}_{\mu}^{j j} T_{\mu}$ into (4.12). It follows from (C.10)-(C.11) that

$$
\operatorname{tr}\left(T_{\gamma} T_{\alpha} T_{\mu} T_{-\alpha}\right)=N \kappa_{\alpha, \mu}^{2} \delta_{\mu+\gamma}
$$

Therefore,

$$
\sum_{\alpha} \frac{\operatorname{tr}\left(\mathcal{S}^{i i} T_{\alpha} \mathcal{S}^{j j} T_{-\alpha}\right)}{N^{2}} E_{2}\left(\omega_{\alpha}+\frac{q_{i j}}{N}\right)=\frac{1}{N} \sum_{\alpha, \mu} \mathcal{S}_{-\mu}^{i i} \mathcal{S}_{\mu}^{j j} E_{2}\left(\omega_{\alpha}+\frac{q_{i j}}{N}\right) \kappa_{\alpha, \mu}^{2}
$$

Using (C.20)-(C.21) and summing up over $\alpha$ we obtain the last term in (4.10).

\subsection{Trigonometric models}

The general classification of the unitary trigonometric $R$-matrices satisfying associative Yang-Baxter equation was given in $[58,59]$. It includes the 7 -vertex deformation [20] of the 6 -vertex $R$-matrix and its $\mathrm{GL}_{N}$ generalizations such as the non-standard $R$-matrix [2]. The integrable tops and related structures based on these $R$-matrices were described in $[36,37]$.

Here we restrict ourselves to the case $N=2$. The 7 -vertex $R$-matrix is of the following form:

$$
R_{12}^{\hbar}(z)=\left(\begin{array}{cccc}
\operatorname{coth}(z)+\operatorname{coth}(\hbar) & 0 & 0 & 0 \\
0 & \sinh ^{-1}(\hbar) \sinh ^{-1}(z) & 0 \\
0 & \sinh ^{-1}(z) \sinh ^{-1}(\hbar) & 0 \\
C \sinh (z+\hbar) & 0 & 0 & \operatorname{coth}(z)+\operatorname{coth}(\hbar)
\end{array}\right)
$$

where $C$ is a constant. In the limit $C \rightarrow 0$ the lower left-hand corner vanishes and we get the 6 -vertex XXZ $R$-matrix. For the classical $r$-matrix and its derivative $\left(F_{12}^{0}(z)=\partial_{z} r_{12}(z)\right)$ we have

$$
r_{12}(z)=\left(\begin{array}{cccc}
\operatorname{coth}(z) & 0 & 0 & 0 \\
0 & 0 & \sinh ^{-1}(z) & 0 \\
0 & \sinh ^{-1}(z) & 0 & 0 \\
C \sinh (z) & 0 & 0 & \operatorname{coth}(z)
\end{array}\right)
$$


and

$$
F_{12}^{0}(q)=\left(\begin{array}{cccc}
-\frac{1}{\sinh ^{2}(q)} & 0 & 0 & 0 \\
0 & 0 & -\frac{\cosh (q)}{\sinh ^{2}(q)} & 0 \\
0 & -\frac{\cosh (q)}{\sinh ^{2}(q)} & 0 & 0 \\
C \cosh (q) & 0 & 0 & -\frac{1}{\sinh ^{2}(q)}
\end{array}\right)
$$

respectively. The Fourier transformed $F^{0}$ matrix is of the form:

$$
F_{12}^{0}(q) P_{12}=\left(\begin{array}{cccc}
-\frac{1}{\sinh ^{2}(q)} & 0 & 0 & 0 \\
0 & -\frac{\cosh (q)}{\sinh ^{2}(q)} & 0 & 0 \\
0 & 0 & -\frac{\cosh (q)}{\sinh ^{2}(q)} & 0 \\
C \cosh (q) & 0 & 0 & -\frac{1}{\sinh ^{2}(q)}
\end{array}\right)
$$

From the latter matrix using (1.32) we obtain

$$
\begin{aligned}
& \mathcal{U}\left(\mathcal{S}^{i j}, \mathcal{S}^{j i}, q_{i}-q_{j}\right)=\operatorname{tr}_{12}\left(\partial_{q_{i}} r_{21}\left(q_{i j}\right) P_{12} \mathcal{S}_{1}^{i j} \mathcal{S}_{2}^{j i}\right)= \\
& \quad=-\frac{1}{\sinh ^{2}\left(q_{i j}\right)}\left(\mathcal{S}_{11}^{i j} \mathcal{S}_{11}^{j i}+\mathcal{S}_{22}^{i j} \mathcal{S}_{22}^{j i}\right)-\frac{\cosh \left(q_{i j}\right)}{\sinh ^{2}\left(q_{i j}\right)}\left(\mathcal{S}_{11}^{i j} \mathcal{S}_{22}^{j i}+\mathcal{S}_{22}^{i j} \mathcal{S}_{11}^{j i}\right)+C \cosh \left(q_{i j}\right) \mathcal{S}_{12}^{i j} \mathcal{S}_{12}^{j i} .
\end{aligned}
$$

Similarly, using (1.33) and (4.17) we get the potential for the model of interacting tops:

$$
\begin{aligned}
& \mathcal{V}\left(\mathcal{S}^{i i}, \mathcal{S}^{j j}, q_{i}-q_{j}\right)=\operatorname{tr}_{12}\left(\partial_{q_{i}} r_{12}\left(q_{i j}\right) \mathcal{S}_{1}^{i i} \mathcal{S}_{2}^{j j}\right)= \\
& \quad=-\frac{1}{\sinh ^{2}\left(q_{i j}\right)}\left(\mathcal{S}_{11}^{i i} \mathcal{S}_{11}^{j j}+\mathcal{S}_{22}^{i i} \mathcal{S}_{22}^{j j}\right)-\frac{\cosh \left(q_{i j}\right)}{\sinh ^{2}\left(q_{i j}\right)}\left(\mathcal{S}_{12}^{i i} \mathcal{S}_{21}^{j j}+\mathcal{S}_{21}^{i i} \mathcal{S}_{12}^{j j}\right)+C \cosh \left(q_{i j}\right) \mathcal{S}_{12}^{i i} \mathcal{S}_{12}^{j j} .
\end{aligned}
$$

The top Hamiltonian $\mathcal{H}^{\text {top }}\left(\mathcal{S}^{i i}\right)$ entering (1.1) or (1.26) is of the form:

$$
\mathcal{H}^{\text {top }}\left(\mathcal{S}^{i i}\right)=\frac{1}{2}\left(\left(\mathcal{S}_{11}^{i i}\right)^{2}+\left(\mathcal{S}_{22}^{i i}\right)^{2}\right)+C\left(\mathcal{S}_{12}^{i i}\right)^{2} .
$$

\subsection{Rational models}

The rational $R$-matrices satisfying the required properties are represented by the 11-vertex deformation [20] of the 6-vertex XXX (Yang's) $R$-matrix. Its higher rank analogues were derived in [66] and $[40,41]$. As in trigonometric case here we restrict ourselves to the case $N=2$. The 11-vertex $R$-matrix is of the following form:

$$
R_{12}^{\hbar}(z)=\left(\begin{array}{cccc}
\hbar^{-1}+z^{-1} & 0 & 0 & 0 \\
-\hbar-z & \hbar^{-1} & z^{-1} & 0 \\
-\hbar-z & z^{-1} & \hbar^{-1} & 0 \\
-\hbar^{3}-2 z \hbar^{2}-2 \hbar z^{2}-z^{3} & \hbar+z & \hbar+z & \hbar^{-1}+z^{-1}
\end{array}\right)
$$

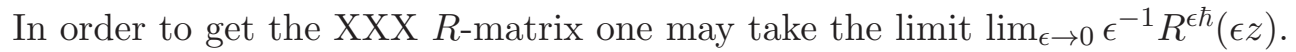


The classical $r$-matrix, the $F_{12}^{0}$ matrix and its Fourier dual are of the form:

$$
\begin{aligned}
r_{12}(z) & =\left(\begin{array}{cccc}
z^{-1} & 0 & 0 & 0 \\
-z & 0 & z^{-1} & 0 \\
-z & z^{-1} & 0 & 0 \\
-z^{3} & z & z & z^{-1}
\end{array}\right) \\
F_{12}^{0}(q) & =\left(\begin{array}{cccc}
-q^{-2} & 0 & 0 & 0 \\
-1 & 0 & -q^{-2} & 0 \\
-1 & -q^{-2} & 0 & 0 \\
-3 q^{2} & 1 & 1 & -q^{-2}
\end{array}\right) \\
F_{12}^{0}(q) P_{12} & =\left(\begin{array}{cccc}
-q^{-2} & 0 & 0 & 0 \\
-1 & -q^{-2} & 0 & 0 \\
-1 & 0 & -q^{-2} & 0 \\
-3 q^{2} & 1 & 1 & -q^{-2}
\end{array}\right)
\end{aligned}
$$

From (4.25) using (1.32) we obtain

$$
\begin{aligned}
\mathcal{U}\left(\mathcal{S}^{i j}, \mathcal{S}^{j i}, q_{i}-q_{j}\right)= & -\frac{1}{\left(q_{i}-q_{j}\right)^{2}}\left(\mathcal{S}_{11}^{i j} \mathcal{S}_{11}^{j i}+\mathcal{S}_{22}^{i j} \mathcal{S}_{22}^{j i}+\mathcal{S}_{11}^{i j} \mathcal{S}_{22}^{j i}+\mathcal{S}_{22}^{i j} \mathcal{S}_{11}^{j i}\right)+ \\
& +\mathcal{S}_{12}^{i j} \mathcal{S}_{22}^{j i}+\mathcal{S}_{22}^{i j} \mathcal{S}_{12}^{j i}-\mathcal{S}_{12}^{i j} \mathcal{S}_{11}^{j i}-\mathcal{S}_{11}^{i j} \mathcal{S}_{12}^{j i}-3\left(q_{i}-q_{j}\right)^{2} \mathcal{S}_{12}^{i j} \mathcal{S}_{12}^{j i}
\end{aligned}
$$

Similarly, from (4.24) using (1.33) we obtain

$$
\begin{aligned}
\mathcal{V}\left(\mathcal{S}^{i i}, \mathcal{S}^{j j}, q_{i}-q_{j}\right)= & -\frac{1}{\left(q_{i}-q_{j}\right)^{2}}\left(\mathcal{S}_{11}^{i i} \mathcal{S}_{11}^{j j}+\mathcal{S}_{22}^{i i} \mathcal{S}_{22}^{j j}+\mathcal{S}_{12}^{i i} \mathcal{S}_{21}^{j j}+\mathcal{S}_{21}^{i i} \mathcal{S}_{12}^{j j}\right)+ \\
& +\mathcal{S}_{12}^{i i} \mathcal{S}_{22}^{j j}+\mathcal{S}_{22}^{i i} \mathcal{S}_{12}^{j j}-\mathcal{S}_{12}^{i i} \mathcal{S}_{11}^{j j}-\mathcal{S}_{11}^{i i} \mathcal{S}_{12}^{j j}-3\left(q_{i}-q_{j}\right)^{2} \mathcal{S}_{12}^{i i} \mathcal{S}_{12}^{j j}
\end{aligned}
$$

The top Hamiltonian $\mathcal{H}^{\text {top }}\left(\mathcal{S}^{i i}\right)$ entering (1.1) or (1.26) is of the form:

$$
\mathcal{H}^{\mathrm{top}}\left(\mathcal{S}^{i i}\right)=\mathcal{S}_{12}^{i i}\left(\mathcal{S}_{22}^{i i}-\mathcal{S}_{11}^{i i}\right) .
$$

\section{Discussion}

A possible application of the obtained family of integrable models is in constructing quantum integrable anisotropic long-rang spin chains. The basic idea is that such spin chains appear from the models of interacting tops by the so-called freezing trick likewise the Haldane-Shastry-Inozemtsev spin chains [31-34] come from the ordinary spin CalogeroMoser-Sutherland models. A direct quantization of the interacting tops is a separate problem, which will be discussed elsewhere. At the same time the quantum Hamiltonian of interacting tops appears in the so-called $R$-matrix-valued Lax pairs for the (classical) spinless Calogero-Moser model [29, 30, 42, 43, 60]. These are the Lax pairs in a large space $\operatorname{Mat}(M, \mathbb{C}) \otimes \operatorname{Mat}(N, \mathbb{C})^{\otimes M}$ :

$$
\mathcal{L}^{\mathrm{CM}}=\sum_{a, b=1}^{M} E_{a b} \otimes \mathcal{L}_{a b}, \quad \mathcal{L}_{a b}=\delta_{a b} p_{a} 1_{N}^{\otimes M}+\nu\left(1-\delta_{a b}\right) R_{a b}^{z}, \quad R_{a b}^{z}=R_{a b}^{z}\left(q_{a}-q_{b}\right) .
$$


and similarly for the accompany $M$-matrix

$$
\mathcal{M}_{a b}^{\mathrm{CM}}=\nu \delta_{a b} d_{a}+\nu\left(1-\delta_{a b}\right) F_{a b}^{z}+\nu \delta_{a b} \mathcal{F}^{0}, \quad F_{a b}^{z}=\partial_{q_{a}} R_{a b}^{z}\left(q_{a}-q_{b}\right),
$$

where

$$
d_{a}=-\sum_{c: c \neq a}^{M} F_{a c}^{0}, \quad \mathcal{F}^{0}=\sum_{b, c: b>c}^{M} F_{b c}^{0}=\sum_{b, c: b>c}^{M} \partial_{q_{b}} r_{b c}\left(q_{b c}\right) .
$$

In the $N=1$ case this Lax pair coincides with the widely known Krichever's result [38] for $\mathrm{gl}_{M}$ Calogero-Moser model. The last term $\mathcal{F}^{0}$ in (5.2) enters $\mathcal{M}$ as a scalar (it is an identity matrix in $\operatorname{Mat}(M, \mathbb{C})$ component $)$ in the auxiliary space $\operatorname{Mat}(M, \mathbb{C})$. Therefore, it can be moved to the l.h.s. of the Lax equation. This yields

$$
\left\{H^{\mathrm{CM}}, \mathcal{L}^{\mathrm{CM}}\right\}+\left[\nu \mathcal{F}^{0}, \mathcal{L}^{\mathrm{CM}}(z)\right]=\left[\mathcal{L}^{\mathrm{CM}}(z), \overline{\mathcal{M}}^{\mathrm{CM}}(z)\right],
$$

where $\overline{\mathcal{M}}^{\mathrm{CM}}=\mathcal{M}^{\mathrm{CM}}-\nu 1_{M} \otimes \mathcal{F}^{0}$. On the one hand (5.4) is just a rewritten classical Lax equation for the spinless Calogero-Moser model. On the other hand we may treat it as half-quantum Lax equation in a sense that the dynamics is given by the interacting tops Hamiltonian (2.60), where the spin variables are already quantized, while the positions and momenta remain classical. Indeed, the quantization of $\mathcal{S}_{1}^{i i}$ in fundamental representation of $\mathrm{GL}_{N}$ is given by the permutation operator $P_{1 j}$. Plugging it into the potential of $(2.60)$ we get the $\mathcal{F}^{0}$ term from (5.2) and (5.4).

Thus the $R$-matrix valued Lax pairs are multidimensional classical Lax pairs for the spinless Calogero-Moser models and at the same time they are quantum Lax pairs for the models of interacting tops with the spin variables being quantized in the fundamental representation of $\mathrm{GL}_{N}$, i.e. the $\mathcal{F}^{0}$ term is the quantization of the potential $\mathcal{V}\left(\mathcal{S}^{i i}, \mathcal{S}^{j j}, q_{i}-\right.$ $\left.q_{j}\right)(1.33)$.

Let us also mention that there is another class of integrable models with the Hamiltonian of type (1.26). These are the Gaudin type models [53]. The corresponding Lax matrix is of size $M \times M$. It has simple poles at $n$ points on elliptic curve (or its degenerations) with the classical spin variables matrices attached to each point. The number of points is not necessarily equal to $M$. It is an interesting task to find interrelations between the Gaudin models and the models of interacting tops through the spectral duality [50-52] based on the rank-size duality transformation.

The classical spinless Calogero-Moser model possesses an equilibrium position, where $p_{i}=0$ and $q_{i}=x_{i}$ (for example, $x_{i}=i / M[21]$ ). At this point the term $\left\{H^{\mathrm{CM}}, \mathcal{L}^{\mathrm{CM}}\right\}$ vanishes from the 1.h.s. of (5.4), and we are left with the quantum Lax equation for some long-range (quantum) spin chain. It is an anisotropic generalization [60] of the HaldaneShastry-Inozemtsev type chains. An open question is which $\mathcal{F}^{0}$ provide integrable spin chains? To confirm integrability we need to construct higher Hamiltonians, which commute with each other and with $\mathcal{F}^{0}\left(q_{i}=x_{i}\right)$. Taking into account all the above we guess that the model of interacting tops together with the freezing trick (the quantum version of the equilibrium position) can be used to calculate higher spin chain Hamiltonians and to prove their commutativity. For this purpose we need to construct a quantization for the model of interacting tops, which is the subject of our next paper. 
Another one intriguing question is to construct relativistic generalization of the models discussed above. While the classical models of relativistic interacting tops are expected to be relatively simple (the block $L^{i j}$ in (2.16) should be replaced by $\operatorname{tr}_{2}\left(\mathcal{S}_{2}^{i j} R_{12}^{z}\left(q_{i j}+\eta\right) P_{12}\right)$ ) its quantum versions and the related long-range spin chain were not studied yet as well as the corresponding $R$-matrix valued Lax pairs.

\section{A Definitions and identities}

The following set of functions is used in this paper [63-65]. The first one is the Kronecker function:

$$
\phi(\eta, z)=\left\{\begin{array}{lll}
1 / \eta+1 / z & - & \text { rational case } \\
\operatorname{coth}(\eta)+\operatorname{coth}(z) & - & \text { trigonometric case } \\
\frac{\vartheta^{\prime}(0) \vartheta(\eta+z)}{\vartheta(\eta) \vartheta(z)} & - & \text { elliptic case }
\end{array}\right.
$$

Its elliptic version is given in terms of the odd theta-function

$$
\vartheta(z)=\sum_{k \in \mathbb{Z}} \exp \left(\pi \imath \tau\left(k+\frac{1}{2}\right)^{2}+2 \pi \imath\left(z+\frac{1}{2}\right)\left(k+\frac{1}{2}\right)\right)
$$

on elliptic curve with moduli $\tau(\operatorname{Im}(\tau)>0)$. Next are the first Eisenstein (odd) function and the Weierstrass (even) $\wp$-function:

$$
E_{1}(z)=\left\{\begin{array}{l}
1 / z, \\
\operatorname{coth}(z), \\
\vartheta^{\prime}(z) / \vartheta(z),
\end{array} \quad \wp(z)=\left\{\begin{array}{l}
1 / z^{2}, \\
1 / \sinh ^{2}(z), \\
-\partial_{z} E_{1}(z)+\frac{1}{3} \frac{\vartheta^{\prime \prime \prime}(0)}{\vartheta^{\prime}(0)} .
\end{array}\right.\right.
$$

We also need the derivatives

$$
E_{2}(z)=-\partial_{z} E_{1}(z)
$$

and

$$
f(z, q) \equiv \partial_{q} \phi(z, q)=\phi(z, q)\left(E_{1}(z+q)-E_{1}(q)\right) .
$$

The one (A.4) is the second Eisenstein function.

The main relation is the Fay trisecant identity:

$$
\phi(z, q) \phi(w, u)=\phi(z-w, q) \phi(w, q+u)+\phi(w-z, u) \phi(z, q+u) .
$$

The following degenerations of (A.6) are necessary for the Lax equations and $r$-matrix structures:

$$
\begin{aligned}
\phi(z, x) f(z, y)-\phi(z, y) f(z, x) & =\phi(z, x+y)(\wp(x)-\wp(y)) \\
\phi(\eta, z) \phi(\eta,-z) & =\wp(\eta)-\wp(z)=E_{2}(\eta)-E_{2}(z) .
\end{aligned}
$$

Also

$$
\begin{aligned}
\phi(z, q) \phi(w, q) & =\phi(z+w, q)\left(E_{1}(z)+E_{1}(w)+E_{1}(q)-E_{1}(z+w+q)\right)= \\
& =\phi(z+w, q)\left(E_{1}(z)+E_{1}(w)\right)-f(z+w, q) .
\end{aligned}
$$


The local behavior of the Kronecker function and the first Eisenstein function near its simple pole at $z=0$ is as follows:

$$
\begin{aligned}
\phi(z, u) & =\frac{1}{z}+E_{1}(u)+\frac{z}{2}\left(E_{1}^{2}(u)-\wp(u)\right)+O\left(z^{2}\right), \\
E_{1}(z) & =\frac{1}{z}+\frac{z}{3} \frac{\vartheta^{\prime \prime \prime}(0)}{\vartheta^{\prime}(0)}+O\left(z^{3}\right) .
\end{aligned}
$$

From (A.10) and (A.5) it follows that

$$
f(0, u)=-E_{2}(u) .
$$

\section{B Spin $\mathrm{gl}_{M}$ Calogero-Moser model}

The Lax equations

$$
\dot{L}^{\operatorname{spin}}(z)=\left[L^{\operatorname{spin}}(z), M^{\text {spin }}(z)\right]
$$

with the Lax pair

$$
\begin{aligned}
L_{i j}^{\mathrm{spin}}(z) & =\delta_{i j}\left(p_{i}+S_{i i} E_{1}(z)\right)+\left(1-\delta_{i j}\right) S_{i j} \phi\left(z, q_{i j}\right), \\
M^{\mathrm{spin}}(z)_{i j} & =\left(1-\delta_{i j}\right) S_{i j} f\left(z, q_{i}-q_{j}\right) .
\end{aligned}
$$

provide (after restriction on the constraints (1.17)) equations of motion

$$
\begin{aligned}
\dot{q}_{i}=p_{i}, \quad \ddot{q}_{i} & =\sum_{j \neq i}^{M} S_{i j} S_{j i} \wp^{\prime}\left(q_{i}-q_{j}\right), \\
\dot{S}_{i i}=0, \quad \dot{S}_{i j} & =\sum_{k \neq i, j}^{M} S_{i k} S_{k j}\left(\wp\left(q_{i}-q_{k}\right)-\wp\left(q_{j}-q_{k}\right)\right), i \neq j .
\end{aligned}
$$

The 1.h.s. of the Lax equations (B.1) is generated by the Hamiltonian (1.16)

$$
\dot{L}^{\text {spin }}(z)=\left\{H^{\text {spin }}, L^{\text {spin }}(z)\right\}
$$

and the linear Poisson-Lie brackets on $\mathrm{gl}_{M}^{*}$ :

$$
\left\{S_{i j}, S_{k l}\right\}=-S_{i l} \delta_{k j}+S_{k j} \delta_{i l} \quad \text { or } \quad\left\{S_{1}, S_{2}\right\}=\left[S_{2}, P_{12}\right] .
$$

Recall that the Poisson reduction with respect to Cartan action (1.18) is non-trivial. For instance, in the rank 1 case (1.22) such reduction leads to the spinless model (1.23). Explicit expression of the reduced Poisson structure depends on a choice of gauge fixation conditions. The equations of motion (B.4)-(B.5) are not the reduced. They are obtained by a simple restriction. To get the final equations one should perform the Dirac reduction and evaluate the Dirac terms.

The classical $r$-matrix structure is as follows:

$$
\begin{array}{r}
\left\{L_{1}^{\mathrm{spin}}(z), L_{2}^{\mathrm{spin}}(w)\right\}=\left[L_{1}^{\mathrm{spin}}(z), r_{12}^{\mathrm{spin}}(z, w)\right]-\left[L_{2}^{\mathrm{spin}}(w), r_{21}^{\mathrm{spin}}(w, z)\right]- \\
-\sum_{i \neq j} E_{i j} \otimes E_{j i}\left(S_{i i}-S_{j j}\right) f\left(z-w, q_{i j}\right)
\end{array}
$$


with

$$
r_{12}^{\mathrm{spin}}(z, w)=E_{1}(z-w) \sum_{i=1}^{M} E_{i i} \otimes E_{i i}+\sum_{i \neq j}^{M} \phi\left(z-w, q_{i j}\right) E_{i j} \otimes E_{j i} .
$$

Here the linear Poisson brackets (B.7) are assumed as well. The Dirac reduction is not yet performed. However, we can see that the restriction on the constraints (1.17) kills the last term in (B.8), and we are left with the standard linear classical $r$-matrix structure. It is enough for Poisson commutativity

$$
\left\{\operatorname{tr}\left(L^{k}(z)\right), \operatorname{tr}\left(L^{n}(w)\right)\right\}=0, \quad \forall k, n \in \mathbb{Z}_{+}, z, w \in \mathbb{C}
$$

necessary for the Liouville integrability. The proof of (B.8) is direct. It is based on the identities (A.6)-(A.9). Let us write down a few examples of verification of (B.8):

the tensor component $E_{i j} \otimes E_{j k}(i \neq j, j \neq k, k \neq i)$ :

l.h.s. of (B.8):

$$
\left\{L_{i j}^{\mathrm{spin}}(z), L_{j k}^{\mathrm{spin}}(w)\right\}=\left\{S_{i j}, S_{j k}\right\} \phi\left(z, q_{i j}\right) \phi\left(w, q_{j k}\right)=-S_{i k} \phi\left(z, q_{i j}\right) \phi\left(w, q_{j k}\right) .
$$

r.h.s. of (B.8):

$$
S_{i k} \phi\left(z, q_{i k}\right) \phi\left(z-w, q_{k j}\right)+S_{i k} \phi\left(w, q_{i k}\right) \phi\left(w-z, q_{j i}\right) .
$$

Expressions (B.11) and (B.12) coincide due to (A.6).

the tensor component $E_{i i} \otimes E_{i j}(i \neq j)$ :

l.h.s. of (B.8):

$$
\begin{aligned}
\left\{L_{i i}^{\mathrm{spin}}(z), L_{i j}^{\mathrm{spin}}(w)\right\} & =\left\{p_{i}, \phi\left(w, q_{i j}\right)\right\} S_{i j}+\left\{S_{i i}, S_{i j}\right\} E_{1}(z) \phi\left(w, q_{i j}\right)= \\
& =S_{i j} f\left(w, q_{i j}\right)-S_{i j} E_{1}(z) \phi\left(w, q_{i j}\right) .
\end{aligned}
$$

r.h.s. of (B.8):

$$
S_{i j} \phi\left(z, q_{i j}\right) \phi\left(z-w, q_{j i}\right)+S_{i j} E_{1}(w-z) \phi\left(w, q_{i j}\right) .
$$

Expressions (B.13) and (B.14) coincide due to (A.9).

the tensor component $E_{i j} \otimes E_{j i}(i \neq j)$ :

l.h.s. of (B.8):

$$
\begin{aligned}
& \left\{L_{i j}^{\mathrm{spin}}(z), L_{j i}^{\mathrm{spin}}(w)\right\}= \\
& \quad=\left\{S_{i j}, S_{j i}\right\} \phi\left(z, q_{i j}\right) \phi\left(w, q_{j i}\right)=\left(S_{i i}-S_{j j}\right) \phi\left(z, q_{i j}\right) \phi\left(-w, q_{i j}\right) .
\end{aligned}
$$

The last term from the r.h.s. of (B.8) contributes in this component. The r.h.s. of (B.8):

$$
\begin{aligned}
\left(p_{i}+\right. & \left.S_{i i} E_{1}(z)-p_{j}-S_{j j} E_{1}(z)\right) \phi\left(z-w, q_{i j}\right)- \\
& -\left(p_{j}+S_{j j} E_{1}(w)-p_{i}-S_{i i} E_{1}(w)\right) \phi\left(w-z, q_{j i}\right)-\left(S_{i i}-S_{j j}\right) f\left(z-w, q_{i j}\right)= \\
& =\left(S_{i i}-S_{j j}\right)\left(\left(E_{1}(z)-E_{1}(w)\right) \phi\left(z-w, q_{i j}\right)-f\left(z-w, q_{i j}\right)\right) .
\end{aligned}
$$

Expressions (B.15) and (B.16) coincide due to (A.9). 


\section{Integrable $\mathrm{gl}_{N}$ tops}

It was shown in [44] (see also $[40,41]$ ) that the Lax equations

$$
\dot{L}(z, S)=[L(z, S), M(z, S)]
$$

are equivalent to equations

$$
\dot{S}=[S, J(S)]
$$

for the Lax pair

$$
L(z, S)=\operatorname{tr}_{2}\left(r_{12}(z) S_{2}\right), \quad M(z, S)=\operatorname{tr}_{2}\left(m_{12}(z) S_{2}\right), \quad S_{2}=1_{N} \otimes S
$$

and

$$
J(S)=\operatorname{tr}_{2}\left(m_{12}(0) S_{2}\right) .
$$

constructed by means of the coefficients of the (classical limit) expansion (1.9) for an $R$ matrix satisfying the associative Yang-Baxter equation (1.8) and the properties (2.2)-(2.5). The answer (C.3) can be written more explicitly. For

$$
r_{12}(z)=\sum_{i, j, k, l=1}^{N} r_{i j k l}(z) e_{i j} \otimes e_{k l}
$$

(C.3) means

$$
L(z, S)=\sum_{i, j, k, l=1}^{N} r_{i j k l}(z) S_{l k} e_{i j}
$$

since $\operatorname{tr}\left(e_{k l} S\right)=S_{l k}$.

Let us briefly describe how these formulae reproduce the elliptic top from [39]. In the elliptic case we need special matrix basis in $\operatorname{Mat}(N, \mathbb{C})$. Consider the matrices

$$
Q_{k l}=\delta_{k l} \exp \left(\frac{2 \pi \imath}{N} k\right), \quad \Lambda_{k l}=\delta_{k-l+1=0 \bmod N}, \quad Q^{N}=\Lambda^{N}=1_{N} .
$$

Then the basis in $\operatorname{Mat}(N, \mathbb{C})$ is given by the following set:

$$
T_{a}=T_{a_{1} a_{2}}=\exp \left(\frac{\pi \imath}{N} a_{1} a_{2}\right) Q^{a_{1}} \Lambda^{a_{2}}, \quad a=\left(a_{1}, a_{2}\right) \in \mathbb{Z}_{N} \times \mathbb{Z}_{N} .
$$

Since

$$
\exp \left(\frac{2 \pi \imath}{N} a_{1} a_{2}\right) Q^{a_{1}} \Lambda^{a_{2}}=\Lambda^{a_{2}} Q^{a_{1}}
$$

we have

$$
T_{\alpha} T_{\beta}=\kappa_{\alpha, \beta} T_{\alpha+\beta}, \quad \kappa_{\alpha, \beta}=\exp \left(\frac{\pi \imath}{N}\left(\beta_{1} \alpha_{2}-\beta_{2} \alpha_{1}\right)\right),
$$

where $\alpha+\beta=\left(\alpha_{1}+\beta_{1}, \alpha_{2}+\beta_{2}\right)$. The non-degenerate pairing is given by the matrix trace:

$$
\operatorname{tr}\left(T_{\alpha} T_{\beta}\right)=N \delta_{\alpha+\beta}, \quad T_{0}=1_{N} .
$$


Define the set of functions numerated by $a=\left(a_{1}, a_{2}\right) \in \mathbb{Z}_{N} \times \mathbb{Z}_{N}$ :

$$
\varphi_{a}\left(z, \omega_{a}+u\right)=\exp \left(2 \pi \imath \frac{a_{2}}{N} z\right) \phi\left(z, \omega_{a}+u\right), \quad \omega_{a}=\frac{a_{1}+a_{2} \tau}{N}
$$

and introduce notation

$$
f_{a}\left(z, \omega_{a}+u\right)=\exp \left(2 \pi \imath \frac{a_{2}}{N} z\right) f\left(z, \omega_{a}+u\right) .
$$

The Baxter-Belavin $R$-matrix [11-13] satisfying all required properties including the Fourier symmetry (2.6) is of the form:

$$
R_{12}^{B B}(\hbar, z)=\sum_{a \in \mathbb{Z}_{N} \times \mathbb{Z}_{N}} \varphi_{a}\left(z, \hbar+\omega_{a}\right) T_{a} \otimes T_{-a} \in \operatorname{Mat}(N, \mathbb{C})^{\otimes 2} .
$$

This $R$-matrix satisfies required properties but with different normalizations. For example, the Fourier symmetry has form $R_{12}^{B B}(\hbar, z) P_{12}=R_{12}^{B B}(z / N, N \hbar)$ (see the Fourier transformation formulae in [69]). To fulfill all requirements including the normalization (2.5) we consider

$$
R_{12}^{\hbar}(z)=R_{12}^{B B}(\hbar / N, z)=\frac{1}{N} \sum_{a \in \mathbb{Z}_{N} \times \mathbb{Z}_{N}} \varphi_{a}\left(z, \frac{\hbar}{N}+\omega_{a}\right) T_{a} \otimes T_{-a} \in \operatorname{Mat}(N, \mathbb{C})^{\otimes 2} .
$$

The corresponding classical $r$-matrix is as follows

$$
r_{12}(z)=\frac{1}{N} E_{1}(z) 1_{N} \otimes 1_{N}+\frac{1}{N} \sum_{a \neq(0,0)} \varphi_{a}\left(z, \omega_{a}\right) T_{a} \otimes T_{-a} \in \operatorname{Mat}(N, \mathbb{C})^{\otimes 2},
$$

and

$$
m_{12}(z)=\frac{E_{1}^{2}(z)-\wp(z)}{2 N^{2}} 1_{N} \otimes 1_{N}+\frac{1}{N^{2}} \sum_{a \neq(0,0)} f_{a}\left(z, \omega_{a}\right) T_{a} \otimes T_{-a} \in \operatorname{Mat}(N, \mathbb{C})^{\otimes 2} .
$$

Then the formulae for the Lax pair (C.3) reproduce the Lax pair of the elliptic top. It is contained in the Lax pair (4.1)-(4.3) as a diagonal $N \times N$ block.

The derivative of the classical $r$-matrix is obtained through (A.5):

$$
\begin{aligned}
F_{12}^{0}(z)=\partial_{z} r_{12}(z)= & -\frac{1}{N} E_{2}(z) 1_{N} \otimes 1_{N}+ \\
& +\frac{1}{N} \sum_{a \neq(0,0)} \varphi_{a}\left(z, \omega_{a}\right)\left(E_{1}\left(z+\omega_{a}\right)-E_{1}(z)+2 \pi \imath \partial_{\tau} \omega_{\alpha}\right) T_{a} \otimes T_{-a}
\end{aligned}
$$

The Fourier symmetry $R_{12}^{\hbar}(z)=R_{12}^{z}(\hbar) P_{12}$ for the $R$-matrix (C.15) is based on the following set of identities for the functions (C.12):

$$
\frac{1}{N} \sum_{\alpha} \kappa_{\alpha, \gamma}^{2} \varphi_{\alpha}\left(N \hbar, \omega_{\alpha}+\frac{z}{N}\right)=\varphi_{\gamma}\left(z, \omega_{\gamma}+\hbar\right), \quad \forall \gamma \in \mathbb{Z}_{N} \times \mathbb{Z}_{N}
$$


Be degeneration of the latter identities on can deduce (see [69]):

$$
\sum_{\alpha} E_{2}\left(\omega_{\alpha}+q\right)=N^{2} E_{2}(N q)
$$

and for $\gamma \neq 0$

$$
\sum_{\alpha} \kappa_{\alpha, \gamma}^{2} E_{2}\left(\omega_{\alpha}+q\right)=-N^{2} \varphi_{\gamma}\left(N q, \omega_{\gamma}\right)\left(E_{1}\left(N q+\omega_{\gamma}\right)-E_{1}(N q)+2 \pi \imath \partial_{\tau} \omega_{\gamma}\right) .
$$

Conversely,

$$
-E_{2}(q)+\sum_{\alpha} \kappa_{\alpha, \gamma}^{2} \varphi_{\alpha}\left(q, \omega_{\alpha}\right)\left(E_{1}\left(q+\omega_{\alpha}\right)-E_{1}(q)+2 \pi \imath \partial_{\tau} \omega_{\alpha}\right)=-E_{2}\left(\omega_{\gamma}+\frac{q}{N}\right) .
$$

\section{Acknowledgments}

The work was partially supported by RFBR grants 18-01-00926 (all authors) and 19-51$18006 \mathrm{Bolg}_{a}$ (I. Sechin). The research of A. Zotov was also supported in part by the HSE University Basic Research Program, Russian Academic Excellence Project '5-100' and by the Young Russian Mathematics award. A. Zotov is also grateful to Sasha Alexandrov for hospitality at the 2-nd Workshop on integrable systems and applications (May, 2019) in IBS Center for Geometry and Physics (Pohang, Korea), where a part of the results was presented.

Open Access. This article is distributed under the terms of the Creative Commons Attribution License (CC-BY 4.0), which permits any use, distribution and reproduction in any medium, provided the original author(s) and source are credited.

\section{References}

[1] G. Aminov, S. Arthamonov, A. Smirnov and A. Zotov, Rational Top and its Classical R-matrix, J. Phys. A 47 (2014) 305207 [arXiv: 1402.3189] [INSPIRE].

[2] A. Antonov, K. Hasegawa and A. Zabrodin, On trigonometric intertwining vectors and nondynamical $R$ matrix for the Ruijsenaars model, Nucl. Phys. B 503 (1997) 747 [hep-th/9704074] [INSPIRE].

[3] V.I. Arnold, Sur la géométrie différentielle des groupes de Lie de dimension infinie et ses applications à l'hydrodynamique des fluides parfaits, Ann. Inst. Fourier 16 (1966) 319.

[4] L.A. Dikii, Hamiltonian systems connected with the rotation group, Funct. Anal. Appl. 6 (1972) 326.

[5] S.V. Manakov, Note on the integration of Euler's equations of the dynamics of an n-dimensional rigid body, Funct. Anal. Appl. 10 (1976) 328.

[6] A.S. Mishenko, Integral geodesics of a flow on Lie groups, Funct. Anal. Appl. 4 (1970) 232.

[7] A.S. Mishenko and A.T. Fomenko, Euler equation on finite-dimensional Lie groups, Math. USSR Izv. 12 (1978) 371.

[8] E. Billey, J. Avan and O. Babelon, The r matrix structure of the Euler-Calogero-Moser model, Phys. Lett. A 186 (1994) 114 [hep-th/9312042] [INSPIRE]. 
[9] E. Billey, J. Avan and O. Babelon, Exact Yangian symmetry in the classical Euler-Calogero-Moser model, Phys. Lett. A 188 (1994) 263 [hep-th/9401117] [InSPIRE].

[10] I. Krichever, O. Babelon, E. Billey and M. Talon, Spin generalization of the Calogero-Moser system and the Matrix KP equation, Amer. Math. Soc. Transl. 170 (1995) 83.

[11] R.J. Baxter, Partition function of the eight vertex lattice model, Annals Phys. 70 (1972) 193 [INSPIRE].

[12] A.A. Belavin, Dynamical Symmetry of Integrable Quantum Systems, Nucl. Phys. B 180 (1981) 189 [inSPIRE].

[13] M.P. Richey and C.A. Tracy, $\mathbb{Z}_{n}$ Baxter model: Symmetries and the Belavin parametrization, J. Stat. Phys. 42 (1986) 311.

[14] M. Bertola, M. Cafasso and V. Roubtsov, Noncommutative Painlevé Equations and Systems of Calogero Type, Commun. Math. Phys. 363 (2018) 503 [arXiv:1710.00736] [InSPIRE].

[15] F. Calogero, Solution of a three-body problem in one-dimension, J. Math. Phys. 10 (1969) 2191 [inSPIRE].

[16] F. Calogero, Solution of the one-dimensional $N$ body problems with quadratic and/or inversely quadratic pair potentials, J. Math. Phys. 12 (1971) 419 [INSPIRE].

[17] B. Sutherland, Exact results for a quantum many body problem in one-dimension, Phys. Rev. A 4 (1971) 2019 [inSPIRE].

[18] B. Sutherland, Exact results for a quantum many body problem in one-dimension. II, Phys. Rev. A 5 (1972) 1372 [InSPIRE].

[19] J. Moser, Three integrable Hamiltonian systems connnected with isospectral deformations, Adv. Math. 16 (1975) 197 [inSPIRE].

[20] I.V. Cherednik, Relativistically Invariant Quasiclassical Limits of Integrable Two-dimensional Quantum Models, Theor. Math. Phys. 47 (1981) 422 [INSPIRE].

[21] E. Corrigan and R. Sasaki, Quantum versus classical integrability in Calogero-Moser systems, J. Phys. A 35 (2002) 7017 [hep-th/0204039] [INSPIRE].

[22] P. Etingof and O. Schiffmann, Twisted traces of intertwiners for Kac-Moody algebras and classical dynamical r-matrices corresponding to generalized Belavin-Drinfeld triples, Math. Res. Lett. 6 (1999) 593 [math.QA/9908115].

[23] P. Etingof and O. Schiffmann, Lectures on the dynamical Yang-Baxter equations, in Quantum Groups and Lie Theory, London Mathematical Society Lecture Note Series, volume 290, Cambridge University Press, Cambridge U.K. (2001), pp. 89-129 [math.QA/9908064].

[24] L. Feher and B.G. Pusztai, Generalizations of Felder's elliptic dynamical r matrices associated with twisted loop algebras of selfdual Lie algebras, Nucl. Phys. B 621 (2002) 622 [math/0109132] [INSPIRE].

[25] S. Fomin and A.N. Kirillov, Quadratic algebras, Dunkl elements, and Schubert calculus, in Advances in geometry, Progress in Mathematics Series, volume 172, Birkhäuser, Boston Massachusetts (1999), pp. 147-182.

[26] A.N. Kirillov, On Some Quadratic Algebras I $\frac{1}{2}$ : Combinatorics of Dunkl and Gaudin Elements, Schubert, Grothendieck, Fuss-Catalan, Universal Tutte and Reduced Polynomials, SIGMA 12 (2016) 002 [arXiv: 1502.00426]. 
[27] J. Gibbons and T. Hermsen, A generalization of the Calogero-Moser systems, Physica D 11 (1984) 337.

[28] S. Wojciechowski, An integrable marriage of the Euler equations with the Calogero-Moser system, Phys. Lett. A 111 (1985) 101.

[29] A. Grekov and A. Zotov, On R-matrix valued Lax pairs for Calogero-Moser models, J. Phys. A 51 (2018) 315202 [arXiv:1801.00245] [InSPIRE].

[30] A. Zotov, Calogero-Moser Model and R-Matrix Identities, Theor. Math. Phys. 197 (2018) 1755.

[31] F.D.M. Haldane, Exact Jastrow-Gutzwiller resonating valence bond ground state of the spin $1 / 2$ antiferromagnetic Heisenberg chain with $1 / R^{2}$ exchange, Phys. Rev. Lett. 60 (1988) 635 [INSPIRE].

[32] B.S. Shastry, Exact solution of an $S=1 / 2$ Heisenberg antiferromagnetic chain with long ranged interactions, Phys. Rev. Lett. 60 (1988) 639 [INSPIRE].

[33] V.I. Inozemtsev, On the connection between the one-dimensional $S=1 / 2$ Heisenberg chain and Haldane-Shastry model, J. Stat. Phys. 59 (1990) 1143 [INSPIRE].

[34] A.P. Polychronakos, Lattice integrable systems of Haldane-Shastry type, Phys. Rev. Lett. 70 (1993) 2329 [hep-th/9210109] [INSPIRE].

[35] K. Hikami and M. Wadati, Integrable spin-12 particle systems with long-range interactions Phys. Lett. A 173 (1993) 263.

[36] T. Krasnov and A. Zotov, Trigonometric Integrable Tops from Solutions of Associative Yang-Baxter Equation, Ann. Henri Poincaré 20 (2019) 2671 [arXiv: 1812. 04209] [INSPIRE].

[37] T. Krasnov and A. Zotov, Trigonometric Integrable Tops from Solutions of Associative Yang-Baxter Equation, Ann. Henri Poincaré 20 (2019) 2671 [arXiv: 1812. 04209] [INSPIRE].

[38] I. Krichever, Elliptic solutions of the Kadomtsev-Petviashvili equation and integrable systems of particles, Funct. Anal. Appl. 14 (1980) 282.

[39] A. Levin, M. Olshanetsky and A. Zotov, Hitchin Systems - Symplectic Hecke Correspondence and Two-dimensional Version, Commun. Math. Phys. 236 (2003) 93 [nlin/0110045] [INSPIRE].

[40] A. Levin, M. Olshanetsky and A. Zotov, Relativistic Classical Integrable Tops and Quantum R-matrices, JHEP 07 (2014) 012 [arXiv: 1405.7523] [INSPIRE].

[41] A. Levin, M. Olshanetsky and A. Zotov, Classical integrable systems and soliton equations related to eleven-vertex R-matrix, Nucl. Phys. B 887 (2014) 400 [arXiv:1406.2995] [INSPIRE].

[42] A. Levin, M. Olshanetsky and A. Zotov, Planck Constant as Spectral Parameter in Integrable Systems and KZB Equations, JHEP 10 (2014) 109 [arXiv:1408.6246] [INSPIRE].

[43] A. Levin, M. Olshanetsky and A. Zotov, Quantum Baxter-Belavin R-matrices and multidimensional Lax pairs for Painlevé VI, Theor. Math. Phys. 184 (2015) 924 [arXiv: 1501.07351] [INSPIRE].

[44] A. Levin, M. Olshanetsky and A. Zotov, Noncommutative extensions of elliptic integrable Euler-Arnold tops and Painlevé VI equation, J. Phys. A 49 (2016) 395202 [arXiv: 1603.06101] [INSPIRE]. 
[45] A. Levin, M. Olshanetsky, A. Smirnov and A. Zotov, Characteristic Classes and Integrable Systems. General Construction, Commun. Math. Phys. 316 (2012) 1 [arXiv:1006. 0702] [INSPIRE].

[46] A. Levin, M. Olshanetsky, A. Smirnov and A. Zotov, Calogero-Moser systems for simple Lie groups and characteristic classes of bundles, J. Geom. Phys. 62 (2012) 1810 [InSPIRE].

[47] A. Levin, M. Olshanetsky, A. Smirnov and A. Zotov, Characteristic Classes and Integrable Systems for Simple Lie Groups, arXiv:1007.4127 [INSPIRE].

[48] A. Levin, M. Olshanetsky, A. Smirnov and A. Zotov, Hecke Transformations of Conformal Blocks in WZW Theory. I. KZB Equations for Non-trivial Bundles, SIGMA 8 (2012) 095 [arXiv:1207.4386] [INSPIRE].

[49] A. Levin, M. Olshanetsky, A. Smirnov and A. Zotov, Characteristic Classes of SL(N)-Bundles and Quantum Dynamical Elliptic R-Matrices, J. Phys. A 46 (2013) 035201 [arXiv: 1208.5750] [INSPIRE].

[50] A. Mironov, A. Morozov, Y. Zenkevich and A. Zotov, Spectral Duality in Integrable Systems from AGT Conjecture, JETP Lett. 97 (2013) 45 [arXiv:1204.0913] [INSPIRE].

[51] A. Mironov, A. Morozov, B. Runov, Y. Zenkevich and A. Zotov, Spectral Duality Between Heisenberg Chain and Gaudin Model, Lett. Math. Phys. 103 (2013) 299 [arXiv:1206.6349] [INSPIRE].

[52] A. Mironov, A. Morozov, B. Runov, Y. Zenkevich and A. Zotov, Spectral dualities in XXZ spin chains and five dimensional gauge theories, JHEP 12 (2013) 034 [arXiv:1307.1502] [INSPIRE].

[53] N. Nekrasov, Holomorphic bundles and many body systems, Commun. Math. Phys. 180 (1996) 587 [hep-th/9503157] [INSPIRE].

[54] A. Polishchuk, Classical Yang-Baxter equation and the $A^{\infty}$-constraint, Adv. Math. 168 (2002) 56 .

[55] A.P. Polychronakos, Calogero-Moser models with noncommutative spin interactions, Phys. Rev. Lett. 89 (2002) 126403 [hep-th/0112141] [INSPIRE].

[56] A.P. Polychronakos, Generalized Calogero models through reductions by discrete symmetries, Nucl. Phys. B 543 (1999) 485 [hep-th/9810211] [INSPIRE].

[57] A.P. Polychronakos, Physics and Mathematics of Calogero particles, J. Phys. A 39 (2006) 12793 [hep-th/0607033] [InSPIRE].

[58] T. Schedler, Trigonometric solutions of the associative Yang-Baxter equation, Math. Res. Lett. 10 (2003) 301 [math.QA/0212258].

[59] A. Polishchuk, Massey products on cycles of projective lines and trigonometric solutions of the Yang-Baxter equations, in Algebra, Arithmetic, and Geometry, Progress in Mathematics Series, volume 270, Birkhäuser, Boston Massachusetts U.S.A. (2010), pp. 573-617 [math. QA/0612761].

[60] I. Sechin and A. Zotov, R-matrix-valued Lax pairs and long-range spin chains, Phys. Lett. B 781 (2018) 1 [arXiv:1801.08908] [INSPIRE].

[61] E.K. Sklyanin, Some algebraic structures connected with the Yang-Baxter equation, Fuct. Anal. Appl. 16 (1982) 263. 
[62] A.G. Reyman and M.A. Semenov-Tian-Shansky, Lie algebras and Lax equations with spectral parameter on an elliptic curve, Zap. Nauchn. Semin. LOMI 150 (1986) 104.

[63] A. Weil, Elliptic functions according to Eisenstein and Kronecker, Springer-Verlag (1976).

[64] D. Mumford, Tata Lectures on Theta I, Birkhäuser, Boston Massachusetts U.S.A. (1983).

[65] D. Mumford, Tata Lectures on Theta II. Jacobian theta functions and differential equations, Birkhäuser, Boston Massachusetts U.S.A. (1984).

[66] A. Smirnov, Degenerate Sklyanin algebras, Central Eur. J. Phys. 8 (2010) 542 [arXiv:0903.1466] [INSPIRE].

[67] A. Zotov and A. Levin, Integrable Model of Interacting Elliptic Tops, Theor. Math. Phys. 146 (2006) 45 [INSPIRE].

[68] A. Zotov and A. Smirnov, Modifications of bundles, elliptic integrable systems, and related problems, Theor. Math. Phys. 177 (2013) 1281.

[69] A. Zotov, Relativistic elliptic matrix tops and finite Fourier transformations, Mod. Phys. Lett. A 32 (2017) 1750169 [arXiv:1706.05601] [INSPIRE]. 